\title{
Test of gravitational red-shift based on tri-frequency combination of microwave frequency links
}

\author{
Xiao Sun $^{1,2}$ (1), Wen-Bin Shen ${ }^{1,2, a}$, Ziyu Shen ${ }^{3}$, Chenghui Cai ${ }^{1}$, Wei Xu ${ }^{1}$, Pengfei Zhang ${ }^{1}$ \\ ${ }^{1}$ Department of Geophysics, School of Geodesy and Geomatics/Key Laboratory of Geospace Environment and Geodesy of Ministry of Education, \\ Wuhan University, Wuhan, China \\ ${ }^{2}$ State Key Laboratory of Information Engineering in Surveying, Mapping and Remote Sensing, Wuhan University, Wuhan, China \\ ${ }^{3}$ Hubei University of Science and Technology, Xianning, China
}

Received: 8 February 2021 / Accepted: 4 July 2021 / Published online: 20 July 2021

(C) The Author(s) 2021

\begin{abstract}
Over the decades, testing gravitational red-shift (GRS) based on microwave links has made great process, including the GPA experiment, the planned Atomic Clock Ensemble in Space mission, and the China Space Station (CSS). Until now, the formulations of microwave links are almost all based on the time comparison. However, there are advantages of using frequency comparison instead of time comparison to test GRS. Here we develop a tri-frequency combination method based on the measurements of the frequency shifts of three independent microwave links between a space station and a ground station. Aiming at the frequency links' accuracy of $3 \times 10^{-16}$, we should consider various effects, including the Doppler effect, second-order Doppler effect, atmospheric frequency shift, tidal effects, refraction caused by the atmosphere, and Shapiro effect, with accuracy levels of tens of centimeters. The CSS will complete construction in 2022, and the formulation proposed in this study will enable us to test GRS at an accuracy level of at least $2 \times 10^{-6}$, which is one order higher than the present accuracy level of $7 \times 10^{-5}$.
\end{abstract}

\section{Introduction}

General relativity theory (GRT) [1] has three classic predictions: Mercury precession, light deflection and gravitational red-shift (GRS). The first and second ones have been confirmed respectively by Einstein himself [1] and a group led by Eddington [2], but the GRS was not tested until 1960.

The first direct experimental verifications of GRS are the series of Pound-Rebka-Snider experiments during 19601965 [3,4], who observed the shift using a Mössbauer emitter and absorber at the Jefferson Physical Laboratory tower

a e-mail: wbshen@sgg.whu.edu.cn (corresponding author) at Harvard University. Later, there is an around-the-world experiment. Four cesium beam clocks were used to fly around the world on commercial jet flights during several days in October 1971, and they flew in opposite directions while recording the time differences [5]. Additionally, other types of experiments measure the shift of spectral lines in the Sun's gravitational field since 1960 [6]. A solar redshift experiment carried out with the Galileo space probe tested the GRS to $1 \%$ accuracy [7]. The most famous test was obtained by the Gravity Probe A (GPA) mission in June 1976, which launched a hydrogen maser onboard a sounding rocket to a height of $10,000 \mathrm{~km}$ [8]. During its flight, frequency comparisons were conducted between the maser on the rocket and a corresponding maser on the ground. The uncertainty on the measurement of GRT is $7 \times 10^{-5}$ [9]. Until now, the most precise indirect tests were performed by eccentric Galileo satellites. The tests were based on the satellites GSAT-0201 and GSAT-0202 of the European Global Navigation Satellite System (GNSS) Galileo, which were accidentally delivered on elliptic instead of circular orbits. Two research teams simultaneously published papers with precision of $(0.19 \pm 2.48) \times 10^{-5}[10]$ and $(4.5 \pm 3.1) \times 10^{-5}[11]$.

To further improve the precision of testing GRS, scientists can obtain benefits from some space missions, such as the Atomic Clock Ensemble in Space (ACES), the SpaceTime Explorer and QUantum Equivalence Space Test (STEQUEST) [12], China Space Station (CSS) mission.

The ACES experiment [13-16], which will be installed onboard the International Space Station (ISS), is an ESACNES mission mainly planned to test the GRS. Equipped with atomic clocks of fractional frequency instability and inaccuracy of $(1-3) \times 10^{-16}$, it aims to test the GRS at a level of $2 \times 10^{-6}[14,15]$, which is one and a half orders of magnitude higher than the GPA experiment. The main onboard instruments are an active hydrogen maser (SHM) and a 
cold cesium atomic clock (PHARAO). The PHARAO clock reaches a fractional frequency stability of $1.1 \times 10^{-13} \sqrt{\tau}$, where $\tau$ is the integration time in seconds, and an accuracy of a few parts in $10^{16}$ [15]. Meanwhile, SHM demonstrates a fractional frequency instability of $1.5 \times 10^{-15}$ after $10,000 \mathrm{~s}$ of integration time. ACES enables frequency/time comparisons between ISS and ground stations by using two kinds of independent time $\&$ frequency transfer links (Microwave Links (MWL) and European Laser Timing (ELT) optical link) to test GRT and develop various applications, for instance in geodesy and time \& frequency metrology $[14,15]$. These science objectives are closely related to the MWL performance [17], and its performance plays a key role in this study. MWL uses the one uplink and two downlinks to transfer time and frequency and will perform with time deviation better than 0.3 ps at $300 \mathrm{~s}, 7$ ps at 1 day, and 23 ps at 10 days of integration time [18].

Similar with ACES mission, CSS will also be equipped with an active hydrogen maser, a cold microwave atom clock and an optical atomic clock, being able to transmit microwave links and optical links. The relevant frequency parameters related to the hydrogen maser and the cold microwave atom clock are at the same level with those of ACES, but the frequency stability of the optical atomic clock will be $8 \times 10^{-18} /$ day $[19,20]$, which will greatly improve the accuracy of testing GRS. Since CSS is under construction and the detailed report of CSS payloads have not been officially released, our paper will focus on general performances of ACES payloads and MWL, which could be applied to CSS time and frequency experiments.

Concerning the ACES mission, some studies have addressed the test of GRS based on time comparison [17,21,22], but there are few publications related to the frequency comparison. Frequency links and comparisons will be available on CSS mission. Compared with time comparison, frequency comparison has the following advantages: (1) it will not be influenced by phase ambiguity because the latter is only relevant in ranging but not in frequency comparison measurements; (2) it can determine the gravitational potential within a short time interval, while for time comparison, we must accumulate data to solve the time changing rate to deduce the GRS value. However, the accuracy of measuring the short-term frequency is largely constrained, which implies that we must also accumulate observations to obtain results with higher accuracy.

In this study, we propose a new formulation to obtain the gravitational potential difference between a space station and a ground station by combining three observed frequencies, referred to as tri-frequency combination (TFC). For the oneway frequency transfer model with a precision requirement of $10^{-16}$, we adopt a formulation accurate to $c^{-3}$ order in free space without medium, which was proposed by [23]. For our theoretical contributions, we extended the model of [23] from free space (vacuum) to real space with media (see Sect. 2 and Appendix A) and formulated the approach to eliminate the first-order Doppler frequency shift (simply Doppler frequency shift hereafter) with considering the time offset among three links (see Sect. 3 and Appendix B). Our final TFC model can successfully eliminate all types of frequency shifts to the order of $10^{-16}$. To verify our model and analyze the demanded magnitude of parameters, we designed simulation experiments considering the real orbit, reliable clocks noises, real atmosphere and real gravity (see Sect. 5).

\section{One-way frequency transfer between space and ground station}

Since we are unknown of the exact carrier frequencies of the CSS microwave links, we formulate the model based on ACES MWLs. The ACES mission uses two different antennas: one Ku-band antenna for uplink and downlink and one S-band antenna for only downward signals. It uses the uplink of carrier frequency $13.475 \mathrm{GHz}$ (Ku band, and the frequency shift will be broadcast to the ground station afterwards) and downlinks of carrier frequency $14.70333 \mathrm{GHz}$ (Ku band) and $2248 \mathrm{MHz}$ (S band) [13], denoted respectively by $f_{1}$, $f_{2}$, and $f_{3}$ throughout this study. The testing precision (will be demonstrated in Sect. 4) is $2 \times 10^{-6}$; thus, we need a frequency transfer model to the level of $1 \times 10^{-16}$, which requires a relativistic model to the order of $c^{-3}$.

First, we consider a downlink from space station A to ground station $\mathrm{B}$. The frequency transfer ratio $f_{\mathrm{A}} / f_{\mathrm{B}}$ between proper frequencies $f_{\mathrm{A}}$ and $f_{\mathrm{B}}$ is determined by the clocks at $\mathrm{A}$ and $\mathrm{B}$. In practice, this is achieved using the transmission of photons from A to B, expressed as [23]

$\frac{f_{\mathrm{A}}}{f_{\mathrm{B}}}=\left(\frac{f_{\mathrm{A}}}{v_{\mathrm{A}}}\right)\left(\frac{v_{\mathrm{A}}}{v_{\mathrm{B}}}\right)\left(\frac{v_{\mathrm{B}}}{f_{\mathrm{B}}}\right)$

where $v_{\mathrm{A}}$ and $v_{\mathrm{B}}$ are the proper frequencies of the photon at $\mathrm{A}$ and $\mathrm{B}$. In a general relativistic framework, the proper frequency shift (in this paper, all frequency shifts are fractional) of the photon from A to B is expressed by [23]

$$
\frac{v_{\mathrm{B}}}{v_{\mathrm{A}}}=\frac{1-\frac{1}{c^{2}}\left[U_{\mathrm{E}}\left(\mathbf{r}_{\mathrm{A}}\right)+\frac{v_{\mathrm{A}}^{2}}{2}\right]}{1-\frac{1}{c^{2}}\left[U_{\mathrm{E}}\left(\mathbf{r}_{\mathrm{B}}\right)+\frac{v_{\mathrm{B}}^{2}}{2}\right]} \frac{q_{\mathrm{B}}}{q_{\mathrm{A}}} .
$$

The first factor on the right-hand side is the sum of the GRS and transverse Doppler frequency shift, and $U_{\mathrm{E}}$ is Newtonian potential of the Earth in the frame of Earth-Centered EarthFixed (ECEF). We denote radial vectors $\mathbf{r}_{\mathrm{A}}=\mathbf{x}_{\mathrm{A}}\left(t_{\mathrm{A}}\right)$ and $\mathbf{r}_{\mathrm{B}}=\mathbf{x}_{\mathrm{B}}\left(t_{\mathrm{B}}\right)$, so $r_{\mathrm{A}}=\left|\mathbf{r}_{\mathrm{A}}\right|, r_{\mathrm{B}}=\left|\mathbf{r}_{\mathrm{B}}\right| \cdot \mathbf{v}_{\mathrm{A}}=\mathbf{v}_{\mathrm{A}}\left(t_{\mathrm{A}}\right)$ and $\mathbf{v}_{\mathrm{B}}=\mathbf{v}_{\mathrm{B}}\left(t_{\mathrm{B}}\right)$ are the coordinate velocities. To the required order of $1 / c^{3}$, the last factor in Eq. (2) is obtained from [23] 


$$
\begin{aligned}
q_{\mathrm{A}}= & 1-\frac{\mathbf{N}_{\mathrm{AB}} \cdot \mathbf{v}_{\mathrm{A}}}{c} \\
& -\frac{4 G M_{\mathrm{E}}}{c^{3}} \frac{\left(r_{\mathrm{A}}+r_{\mathrm{B}}\right) \mathbf{N}_{\mathrm{AB}} \cdot \mathbf{v}_{\mathrm{A}}+R_{\mathrm{AB}} \frac{\mathbf{r}_{\mathrm{A}} \cdot \mathbf{v}_{\mathrm{A}}}{r_{\mathrm{A}}}}{\left(r_{\mathrm{A}}+r_{\mathrm{B}}\right)^{2}-R_{\mathrm{AB}}^{2}} \\
q_{\mathrm{B}}= & 1-\frac{\mathbf{N}_{\mathrm{AB}} \cdot \mathbf{v}_{\mathrm{B}}}{c} \\
& -\frac{4 G M_{\mathrm{E}}}{c^{3}} \frac{\left(r_{\mathrm{A}}+r_{\mathrm{B}}\right) \mathbf{N}_{\mathrm{AB}} \cdot \mathbf{v}_{\mathrm{B}}-R_{\mathrm{AB}} \frac{\mathbf{r}_{\mathrm{B}} \cdot \mathbf{v}_{\mathrm{B}}}{r_{\mathrm{B}}}}{\left(r_{\mathrm{A}}+r_{\mathrm{B}}\right)^{2}-R_{\mathrm{AB}}^{2}}
\end{aligned}
$$

with $\mathbf{R}_{\mathrm{AB}}=\mathbf{r}_{\mathrm{B}}-\mathbf{r}_{\mathrm{A}}, R_{\mathrm{AB}}=\left|\mathbf{R}_{\mathrm{AB}}\right|$, and $\mathbf{N}_{\mathrm{AB}}=\mathbf{R}_{\mathrm{AB}} / R_{\mathrm{AB}}$. The last terms in Eqs. (3) and (4) are caused by curved geometry in the general relativistic framework, referred to as Shapiro effect. To calculate the Shapiro effect, it is accurate enough to take the Earth as a spherically symmetric unform body [23].

Using simplified notations

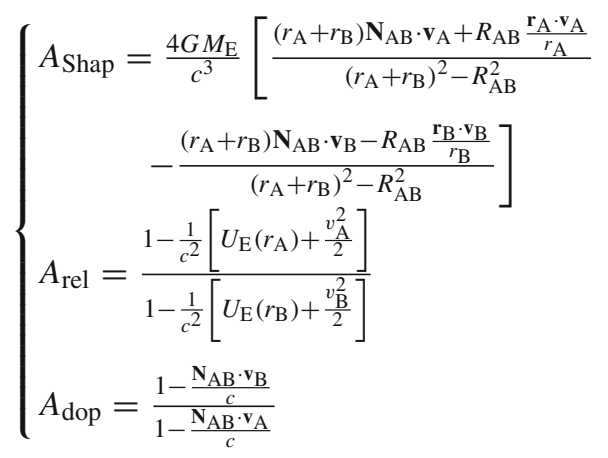

we have

$\frac{\nu_{\mathrm{B}}}{v_{\mathrm{A}}}=A_{\text {rel }}\left(A_{\text {dop }}+A_{\text {Shap }}\right)$.

Equations (5) and (6) hold only in vacuum. In a real space with medium, electromagnetic waves experience a change in direction of propagation or refractive bending when they are transmitted through the atmosphere, which is divided into the troposphere $(0-60 \mathrm{~km})$ and ionosphere $(60-2000 \mathrm{~km})$. Because of the refraction phenomenon, the direction of the refracted ray at the space station slightly differs from the unrefracted line-of-sight direction [24], as Fig. 1 shows. This phenomenon has significant applications in the GPS/MET (Global Positioning System/Meteorology) experiment [25] and will cause a slight change of Doppler effect in Eq. (6). Here, by defining $\bar{A}_{\text {dop }}$ as Doppler frequency considering the atmosphere, we have

$\frac{\nu_{\mathrm{B}}}{v_{\mathrm{A}}}=A_{\text {rel }}\left(\bar{A}_{\text {dop }}+A_{\text {Shap }}\right)$.

The refractive index in the ionosphere is relevant with the carrier frequency, but the refractive index in troposphere is nearly irrelevant with it. Therefore, for all links $f_{1}, f_{2}$ and $f_{3}$, supposing that they are simultaneously emitted, the bending

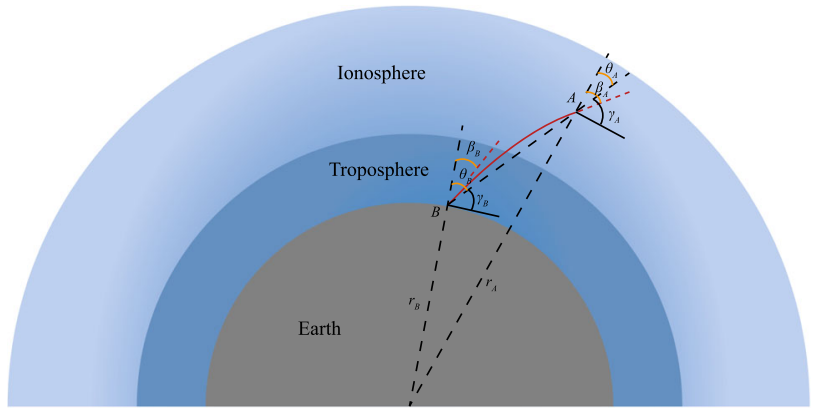

Fig. 1 Principle of ray refraction through the atmosphere for a space station. $r$ is distance from the Earth center, $\beta$ is the angle between the tangent of the electromagnetic wave and the normal of the layer, $\theta$ is the angle between $\mathrm{AB}$ line direction and layer normal, and $\gamma$ is the complementary angle of $\theta$

effects of the troposphere are approximately identical, which makes it easy to wipe out the tropospheric part. For the ionospheric part, to the order of $f^{-2}$, we have $[25,26]$

$n=1-40.3 \frac{n_{\mathrm{e}}}{f^{2}}$

where $n_{\mathrm{e}}$ is the electron density per cubic meter; high orders such as $f^{-3}$ are neglected because they are at least two magnitudes smaller than the order of $f^{-2}$ [27], which we will discuss later.

In the phase form, Doppler frequency shift is given by phase path $P$ of the radio wave [26,28-30]:

$\Delta f_{\mathrm{dop}}=-\frac{f}{c} \frac{d P}{d t}=-\frac{1}{\lambda} \frac{d P}{d t}$

where the velocity of the source induces a change in $\lambda$, and the velocity of the observer changes $d P / d t$. Thus, in vacuum, the first-order Doppler frequency shift can be derived in terms of $A_{\mathrm{dop}}$, as expressed by Eq. (5).

More specifically, we have [31]

$\Delta f_{\text {dop }}=-\frac{1}{\lambda}\left(\int_{\mathrm{A}}^{B} \frac{\partial n}{\partial t} \cos \alpha d s+n_{\mathrm{B}} \mathbf{T}_{\mathrm{B}} \cdot \mathbf{v}_{\mathrm{B}}-n_{\mathrm{A}} \mathbf{T}_{\mathrm{A}} \cdot \mathbf{v}_{\mathrm{A}}\right)$

where $\mathbf{T}$ is the unit vector of the wave normal, $n$ is the refractive index, and $\alpha$ is the angle between the wave normal and the ray direction. Suppose the atmosphere is an isotropic medium, the refractive index is nearly independent of ray directions, and $\alpha=0$ [32]. From Eq. (10), the atmospheric influence can be explained by two reasons: the refractive index varies with time [28,29], and the wave path varies because the observer moves [33].

Due to the velocity of the source, the wavelength of the signal is expressed as

$\lambda=\lambda_{0}\left(1-\frac{n_{\mathrm{A}} \mathbf{T}_{\mathrm{A}} \cdot \mathbf{v}_{\mathrm{A}}}{c}\right)$. 
Considering $\bar{A}_{\text {dop }}$ defined in Eq. (7), with considering Eqs. (10) and (11), we have

$$
\bar{A}_{\text {dop }}=\frac{f+\Delta f_{\text {dop }}}{f}=\frac{1-\frac{n_{\mathrm{B}} \mathbf{T}_{\mathrm{B}} \cdot \mathbf{v}_{\mathrm{B}}}{c}-\int_{\mathrm{A}}^{B} \frac{\partial n}{\partial t} \cos \alpha d s}{1-\frac{n_{\mathrm{A}} \mathbf{T}_{\mathrm{A}} \cdot \mathbf{v}_{\mathrm{A}}}{c}} .
$$

With the height of a space station of approximately $400 \mathrm{~km}$ [13], the space-ground links lie in the middle layer of the ionosphere. The integral term of the refractive index in Eq. (12) can be expressed as the sum of the ionospheric and tropospheric parts, and if setting $\alpha=0$, we have [30]

$$
\begin{aligned}
\int_{\mathrm{A}}^{B} \frac{\partial n}{\partial t} \cos \alpha d s= & -\frac{40.3}{c f^{2}} \frac{d}{d t} \int_{\mathrm{Li}} n_{\mathrm{e}} d s \\
& +\frac{1}{c} \frac{d}{d t} \int_{\mathrm{Lt}}\left(M_{1}+M_{2}\right) d s
\end{aligned}
$$

where $n_{\mathrm{e}}$ is the electron density along the trajectory, $M_{1}=$ $77.6 \times 10^{-6} \mathrm{p} / T$, and $M_{2}=0.373 \varepsilon / T^{2}$ with temperature $T$, total pressure $p$ and partial pressure of water vapor $\varepsilon$ along the trajectory.

For this expansion, we neglect high order terms and rewrite Eq. (12) as

$$
\begin{aligned}
\bar{A}_{\text {dop }}= & \frac{1-\frac{n_{\mathrm{B}} \mathbf{T}_{\mathrm{B}} \cdot \mathbf{v}_{\mathrm{B}}}{c}}{1-\frac{n_{\mathrm{A}} \mathbf{T}_{\mathrm{A}} \cdot \mathbf{v}_{\mathrm{A}}}{c}} \\
& +\frac{40.3}{c f^{2}} \frac{d}{d t} \int_{\mathrm{Li}} n_{\mathrm{e}} d s-\frac{1}{c} \frac{d}{d t} \int_{\mathrm{Lt}}\left(M_{1}+M_{2}\right) d s
\end{aligned}
$$

where (referring to Appendix A)

$$
\begin{aligned}
\frac{1-\frac{n_{\mathrm{B}} \mathbf{T}_{\mathrm{B}} \cdot \mathbf{v}_{\mathrm{B}}}{c}}{1-\frac{n_{\mathrm{A}} \mathbf{T}_{\mathrm{A}} \cdot \mathbf{v}_{\mathrm{A}}}{c}}=\left[1-\frac{\mathbf{N}_{\mathrm{AB}} \cdot \mathbf{v}_{\mathrm{B}}}{c}+\frac{v_{\mathrm{B} x} \delta_{\mathrm{B}} \sin \gamma_{\mathrm{B}}-v_{\mathrm{By}} \delta_{\mathrm{B}} \cos \gamma_{\mathrm{B}}}{c}\right. \\
\left.-\frac{\left(M_{1}+M_{2}\right) \mathbf{N}_{\mathrm{AB}} \cdot \mathbf{v}_{\mathrm{B}}}{c}\right] /\left[1-\frac{\mathbf{N}_{\mathrm{AB}} \cdot \mathbf{v}_{\mathrm{A}}}{c}\right. \\
\left.-\frac{v_{\mathrm{Ax}} \delta_{\mathrm{A}} \sin \gamma_{\mathrm{B}}-v_{\mathrm{Ay}} \delta_{\mathrm{A}} \cos \gamma_{\mathrm{B}}}{c}+\frac{40.3 n_{\mathrm{e}} \mathbf{N}_{\mathrm{AB}} \cdot \mathbf{v}_{\mathrm{A}}}{c f^{2}}\right]
\end{aligned}
$$

where $v_{\mathrm{Ax}}, v_{\mathrm{Ay}}, v_{\mathrm{Bx}}$, and $v_{\mathrm{By}}$ are components of velocities $\mathbf{v}_{\mathrm{A}}$ and $\mathbf{v}_{\mathrm{B}}$ of space station $\mathrm{A}$ and ground site $\mathrm{B}$ projected in the refraction plane in Fig. 1, and $\delta_{\mathrm{A}}$ and $\delta_{\mathrm{B}}$ are deviated angles from the line of sight. More details are referred to Appendix A.

In summary, focusing on the effect of the variation of the refractive index and wave path, we can obtain the expression of $\bar{A}_{\text {dop. }}$. Then, the following effects are separated from each other: the refraction part (not time-varying parts), ionospheric and tropospheric frequency shift (time-varying parts), and Shapiro effect. Thus, the one-way frequency transfer model can be written as

$\frac{\nu_{\mathrm{B}}}{v_{\mathrm{A}}}=A_{\text {rel }}\left(A_{\text {dop }}+\delta f_{\text {refr }}+\delta f_{\text {ion }}+\delta f_{\text {trop }}+A_{\text {Shap }}\right)$

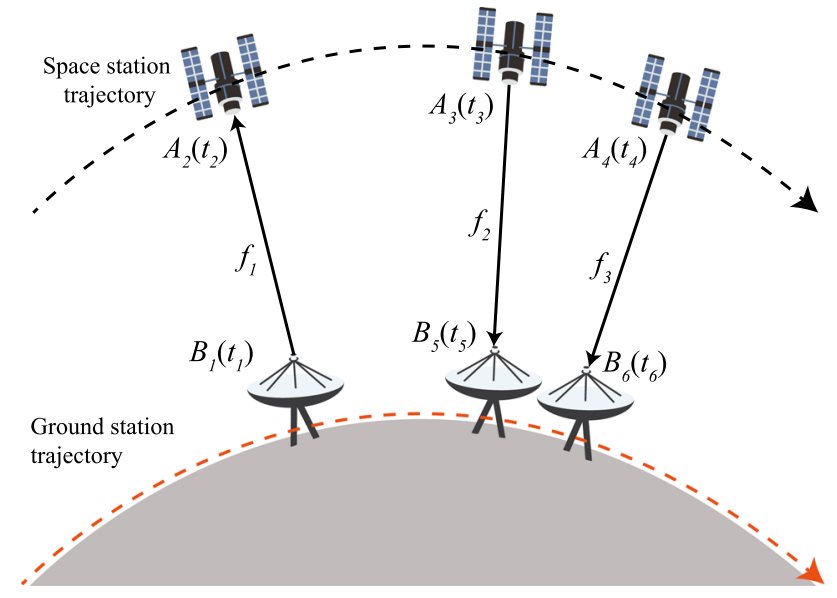

Fig. 2 MWL principle (modified after [34]). At time $t_{1}$, the ground station emits signal $f_{1}$ to the space station, which is received at time $t_{2}$. Meanwhile, two signals $f_{2}$ and $f_{3}$ are emitted to the ground station at time $t_{3}$ and $t_{4}$ (they are approximately equal to $t_{2}$ ), which are received at time $t_{5}$ and $t_{6}$

where

$$
\begin{aligned}
\delta f_{\text {refr }}= & \frac{\left(v_{\mathrm{Ax}} \delta_{\mathrm{A}}+v_{\mathrm{Bx}} \delta_{\mathrm{B}}\right) \sin \gamma_{\mathrm{B}}-\left(v_{\mathrm{Ay}} \delta_{\mathrm{A}}+v_{\mathrm{By}} \delta_{\mathrm{B}}\right) \cos \gamma_{\mathrm{B}}}{c} \\
& -\frac{\left(M_{1}+M_{2}\right) \mathbf{N}_{\mathrm{AB}} \cdot \mathbf{v}_{\mathrm{B}}}{c}-\frac{40.3 n_{\mathrm{e}} \mathbf{N}_{\mathrm{AB}} \cdot \mathbf{v}_{\mathrm{A}}}{c f^{2}} \\
\delta f_{\text {ion }}= & \frac{40.3}{c f^{2}} \frac{d}{d t} \int_{\mathrm{Li}} n_{\mathrm{e}} d s \\
\delta f_{\text {trop }}= & -\frac{1}{c} \frac{d}{d t} \int_{\mathrm{Lt}}\left(M_{1}+M_{2}\right) d s
\end{aligned}
$$

where $A_{\text {rel }}, A_{\text {dop }}, A_{\text {Shap }}$ are defined in Eq. (5), $\delta f_{\text {refr }}$ is the bending effect on Doppler frequency shift, which is caused by refraction, $\delta f_{\text {ion }}$ and $\delta f_{\text {trop }}$ are atmospheric effects caused by the time-varying refractive index. For the ACES links, estimates show that the magnitude of $\delta f_{\text {dop }}$ is approximately $10^{-5} ; \delta f_{\text {rel }}$ is approximately $10^{-10}$ [23]. The magnitudes of the quantities $\delta f_{\text {ion }}, \delta f_{\text {ion }}$ and $\delta f_{\text {trop }}$ will be investigated in simulations in Sect. 5.

\section{Formulation to test GRS}

\subsection{Tri-frequency combination}

Although all of these links are independent and can be synchronized afterwards by data processing, the synchronization error may cause severe residual errors. In this study, we list coordinate time $t_{1} \sim t_{6}$ to identify six events and use a combination of three frequency links to test the GRS, as Fig. 2 shows. Later, we will analyze the required synchronization precision to test GRS at the $2 \times 10^{-6}$ level.

In our formulation, we use a nonrotating geocentric spacetime coordinate system. When we mention time, it refers to 
coordinate time. At time $t_{1}$, Ku-band signal $f_{1}$ is emitted and received by the space station at time $t_{2}$. Meanwhile, two signals $f_{2}$ and $f_{3}$ are emitted from the space station at time $t_{3}$ and $t_{4}$, respectively, and received by the ground station at time $t_{5}$ and $t_{6}$, respectively. If we define a time interval by $T_{i j}=t_{j}-t_{i}, T_{23}$ and $T_{34}$ will theoretically be synchronized to zero, but in practice, there is a difference between them.

For frequency links $f_{1}=13.475 \mathrm{GHz}, f_{2}=14.70333$ $\mathrm{GHz}$ and $f_{3}=2248 \mathrm{MHz}$ (these values are provided by ACES), the third link is of low frequency, which greatly suffers from ionospheric effects. Suppose $T_{34}$ is extremely small $(<1 \mu \mathrm{s})$, the only different error between link 2 and link 3 is the ionospheric error, because other shifts in link 2 and link 3 are close. We define $f_{1}^{\prime}, f_{2}^{\prime}$ and $f_{3}^{\prime}$ as the received frequencies corresponding to emitted frequencies $f_{1}, f_{2}$ and $f_{3}$.

If we divide frequency shift $f_{2}^{\prime} / f_{2}$ by frequency shift $f_{3}^{\prime} / f_{3}$, based on Eqs. (5), (16) and (17), the first-order Doppler effect, relativistic effects (including second-order Doppler effects and GRS) and tropospheric effects are cancelled, obtaining

$$
\begin{aligned}
& \frac{f_{2}{ }^{\prime}}{f_{2}} / \frac{f_{3}{ }^{\prime}}{f_{3}}=1+\left(1-\frac{f_{2}^{2}}{f_{3}^{2}}\right)\left[\frac{40.3}{c f_{2}^{2}} \frac{d}{d t} \int_{\mathrm{Li}} n_{\mathrm{e}} d s\right. \\
& +\frac{\left(v_{\mathrm{Ax}} \delta_{\mathrm{A}}^{\text {ion }}+v_{\mathrm{Bx}} \delta_{\mathrm{B}}^{\text {ion }}\right) \sin \gamma_{\mathrm{B}}}{c} \\
& \left.-\frac{\left(v_{\mathrm{Ay}} \delta_{\mathrm{A}}^{\text {ion }}+v_{\mathrm{By}} \delta_{\mathrm{B}}^{\text {ion }}\right) \cos \gamma_{\mathrm{A}}}{c}+\frac{40.3 n_{\mathrm{e}} \mathbf{N}_{\mathrm{AB}} \cdot \mathbf{v}_{\mathrm{A}}}{c f_{2}^{2}}\right]
\end{aligned}
$$

where $\delta_{\mathrm{A}}^{\text {ion }}$ and $\delta_{\mathrm{B}}^{\text {ion }}$ are the refractive angles inversely proportional to the square of the carrier frequency (see Appendix A). In our simulations, these variables have magnitudes of $10^{-5} \mathrm{rad}$. We note that all terms in the square brackets in Eq. (18) are inversely proportional to the square of the carrier frequency.

Referring to Fig. 2, the ground station has moved a certain distance (approximately 1 meter at present case) from $t_{1}$ to $t_{5}$; thus, the first-order Doppler frequency shift cannot be completely cancelled from Eq. (16). By the same technique, we divide frequency shift $f_{1}^{\prime} / f_{1}$ by frequency shift $f_{2}^{\prime} / f_{2}$, obtaining

$$
\begin{aligned}
& \frac{f_{1}{ }^{\prime}}{f_{1}} / \frac{f_{2}{ }^{\prime}}{f_{2}} \\
& =\frac{m_{1}}{m_{2}} \frac{\left.\frac{1-\frac{1}{c^{2}}\left(U_{\mathrm{B} 1}+\frac{v_{\mathrm{B} 1}^{2}}{2}\right)}{1-\frac{1}{c^{2}}\left(U_{\mathrm{A} 2}+\frac{v_{\mathrm{A} 2}^{2}}{2}\right)}\left(\frac{1-\frac{\mathrm{N}_{\mathrm{B} 1 \mathrm{~A} 2} \cdot \mathrm{v}_{\mathrm{A} 2}}{c}}{1-\frac{\mathrm{N}_{\mathrm{B} 1 \mathrm{~A} 2 \cdot \mathrm{A}_{\mathrm{B} 1}}}{c}}-A_{\text {Shap, link } 1)}\right)\right]}{\left.\frac{1-\frac{1}{c^{2}}\left(U_{\mathrm{A} 3}+\frac{v_{\mathrm{A}}^{2}}{2}\right)}{1-\frac{1}{c^{2}}\left(U_{\mathrm{B} 5}+\frac{v_{\mathrm{B} 5}^{2}}{2}\right)}\left(\frac{1-\frac{\mathrm{N}_{\mathrm{A} 3 \mathrm{~B} 5} \cdot \mathrm{v}_{\mathrm{B} 5}}{c}}{1-\frac{\mathrm{N}_{\mathrm{A} 3 \mathrm{~B} 5} \cdot \mathrm{v}_{\mathrm{A} 3}}{c}}-A_{\text {Shap, link2 }}\right)\right]}
\end{aligned}
$$

where $B i$ is the position of the ground station at time $t_{i}$, and $A i$ is the position of the space station at time $t_{i}$. Here, $m_{1} / m_{2}$ is the solved ionospheric part

$$
\begin{aligned}
\frac{m_{1}}{m_{2}} & =1+\left(\frac{f_{2}^{2}}{f_{1}^{2}}-1\right)\left[\frac{40.3}{c f_{2}^{2}} \frac{d}{d t} \int_{\mathrm{Li}} \frac{d}{d t} n_{\mathrm{e}} d s\right. \\
& +\frac{\left(v_{\mathrm{Ax}} \delta_{\mathrm{A}}^{\text {ion }}+v_{\mathrm{Bx}} \delta_{\mathrm{B}}^{\text {ion }}\right) \sin \gamma_{\mathrm{B}}}{c} \\
& \left.-\frac{\left(v_{\mathrm{Ay}} \delta_{\mathrm{A}}^{\text {ion }}+v_{\mathrm{By}} \delta_{\mathrm{B}}^{\text {ion }}\right) \cos \gamma_{\mathrm{A}}}{c}+\frac{40.3 n_{\mathrm{e}} \mathbf{N}_{\mathrm{AB}} \cdot \mathbf{v}_{\mathrm{A}}}{c f_{2}^{2}}\right]
\end{aligned}
$$

where the terms in the square brackets appeared in Eq. (18) are related to ionospheric effects. Combining Eqs. (18) and (20) gives rise to the following relation

$\frac{m_{1}}{m_{2}}=1+\left(\frac{f_{2}^{2}}{f_{1}^{2}}-1\right)\left(\frac{f_{3}^{2}}{f_{3}^{2}-f_{2}^{2}}\right)\left(\frac{f_{2}{ }^{\prime}}{f_{2}} / \frac{f_{3}{ }^{\prime}}{f_{3}}-1\right)$.

If the difference in space parameters of the space station and ground station at different time points is ignored, the Doppler effect in Eq. (19) can be directly eliminated. However, at different time points such as $t_{2}$ and $t_{3}$, spatial parameters of the space station will be moderately different, which results in Doppler residuals in Eq. (19). According to Appendix B and accurate to the order of $c^{-3}$, we have

$$
\begin{aligned}
& \frac{f_{1}{ }^{\prime}}{f_{1}} / \frac{f_{2}{ }^{\prime}}{f_{2}}=\frac{m_{1}}{m_{2}}\left\{\left[1-\left(\frac{\mathbf{N}_{\mathrm{A} 3 \mathrm{~B} 5} \cdot \mathbf{v}_{\mathrm{A} 3}}{c}\right)^{2}\right.\right. \\
& \left.+\frac{2\left(\mathbf{N}_{\mathrm{A} 3 \mathrm{~B} 5} \cdot \mathbf{v}_{\mathrm{A} 3}\right)\left(\mathbf{N}_{\mathrm{A} 3 \mathrm{~B} 5} \cdot \mathbf{v}_{\mathrm{B} 5}\right)}{c^{2}}-\frac{2 \mathbf{v}_{\mathrm{A} 3} \cdot \mathbf{v}_{\mathrm{B} 5}}{c^{2}}+K_{1} T_{23}\right] \\
& /\left[1+\left(\frac{\mathbf{N}_{\mathrm{A} 3 \mathrm{~B} 5} \cdot \mathbf{v}_{\mathrm{B} 5}}{c^{2}}\right)^{2}-\frac{2 v_{\mathrm{B} 5}^{2}}{c^{2}}-\frac{2 \mathbf{R}_{\mathrm{A} 3 \mathrm{~B} 5} \cdot \mathbf{a}_{\mathrm{B} 5}}{c^{2}}+K_{2} T_{23}\right] \\
& \left.-A_{\text {Shap, link } 2}\right\} \cdot\left[1-\frac{1}{c^{2}}\left(U_{\mathrm{B} 5}-U_{\mathrm{A} 3}+\frac{v_{\mathrm{B} 5}^{2}-v_{\mathrm{A} 3}^{2}}{2}\right)\right]^{2} \\
& K_{1}=\frac{\mathbf{N}_{\mathrm{A} 3 \mathrm{~B} 5} \cdot\left(\mathbf{v}_{\mathrm{B} 5}-\mathbf{v}_{\mathrm{A} 3}\right)\left(\mathbf{N}_{\mathrm{A} 3 \mathrm{~B} 5} \cdot \mathbf{v}_{\mathrm{A} 3}\right)}{c R_{\mathrm{A} 3 \mathrm{~B} 5}} \\
& -\frac{\left(\mathbf{v}_{\mathrm{B} 5}-\mathbf{v}_{\mathrm{A} 3}\right) \cdot \mathbf{v}_{\mathrm{A} 3}}{c R_{\mathrm{A} 3 \mathrm{~B} 5}}-\frac{\mathbf{N}_{\mathrm{A} 3 \mathrm{~B} 5} \cdot \mathbf{a}_{\mathrm{A} 3}}{c} \\
& K_{2}=\frac{\mathbf{N}_{\mathrm{A} 3 \mathrm{~B} 5} \cdot\left(\mathbf{v}_{\mathrm{B} 5}-\mathbf{v}_{\mathrm{A} 3}\right)\left(\mathbf{N}_{\mathrm{A} 3 \mathrm{~B} 5} \cdot \mathbf{v}_{\mathrm{B} 5}\right)}{c R_{\mathrm{A} 3 \mathrm{~B} 5}} \\
& -\frac{\left(\mathbf{v}_{\mathrm{B} 5}-\mathbf{v}_{\mathrm{A} 3}\right) \cdot \mathbf{v}_{\mathrm{B} 5}}{c R_{\mathrm{A} 3 \mathrm{~B} 5}}
\end{aligned}
$$

where $m_{1} / m_{2}$ is determined by Eq. (21); $A_{\text {Shap,link2 }}$ is a term of $c^{-3}$; since the magnitudes of $T_{23}$ and $T_{15}$ are at most $c^{-1}$, the difference between $A_{\text {Shap,link1 }}$ and $A_{\text {Shap,link2 }}$ in Eq. (21) is at most $c^{-4}$, which is negligible, as is the difference between $v_{\mathrm{a} 2}^{2} / c^{2}$ and $v_{\mathrm{a} 3}^{2} / c^{2}$.

\subsection{Test of GRS}

In a static gravitational field, suppose that two atomic clocks are located at different positions. Then, we compare their 
frequencies by a certain frequency transfer method. Based on general relativity, the GRS between clocks is proportional to their gravitational potential difference $\Delta U$, expressed as

$\frac{\Delta v}{v}=\frac{\Delta U}{c^{2}}$.

To test the GRS by a standard convention, parameter $\alpha$ is introduced via the following expression [35]

$z=\frac{\Delta v}{v}=(1+\alpha) \frac{\Delta U}{c^{2}}$

where $\alpha$ vanishes when Einstein equivalence principle (EEP) is valid.

In our study, we develop a similar equation using another parameter $\beta$

$z=\Delta U_{\mathrm{m}}=(1+\beta) \Delta U$

where $\Delta U_{\mathrm{m}}$ is the measured gravitational potential difference by Eqs. (22) and (23), and $\Delta U$ is the standard gravitational potential difference developed by the Earth gravity field model. There are testing errors in both $\Delta U_{\mathrm{m}}$ and $\Delta U$, and the corresponding uncertainties should be calculated using the following equation

$u=\sqrt{u_{\Delta} \mathrm{U}_{\mathrm{m}}^{2}+(1+\beta)^{2} u_{\Delta} \mathrm{U}^{2}}$

where $u_{\Delta} \mathrm{U}_{\mathrm{m}}$ and $u_{\Delta} \mathrm{U}$ are the uncertainties of $\Delta U_{\mathrm{m}}$ and $\Delta U$, respectively.

\subsection{Error sources}

Generally, errors are divided into systematic errors and random errors. All of the aforementioned errors are systematic errors, including Doppler frequency shift, atmospheric frequency shift (including ionospheric, tropospheric and refractive frequency shift), relativistic frequency shift and Shapiro frequency shift. These frequency shifts can be eliminated using our TFC model. However, there are still residuals which need to be considered. These residuals and tidal effects will be discussed in Sect. 4.

Random errors are caused by devices (e.g., atomic clocks, cables and emitters) and measurements (e.g., velocities and accelerations in Eqs. (22) and (23)). Clocks suffer from many kinds of noises that can hardly be modelled. Without the noise components of the space clocks, we can only simulate the clock data to approach the true performance. For measurement noises, the accuracy for parameters $\mathbf{r}_{\mathrm{A}}, \mathbf{v}_{\mathrm{A}}, \mathbf{a}_{\mathrm{A}}$ and $T_{23}$ must be carefully controlled. In Sect. 5 we will discuss the parameter demands using simulated observations.

\section{Residual errors}

Although Sect. 3.1 provides a practical calculation model to test GRS using the TFC method, there are residual errors due to some kinds of approximations in the model derivation. In addition, for the purpose of this study, we did not consider the influences caused by planets.

The model of the TFC method can be summarized by Eqs. (21)-(23). In step one, frequency shifts of downlinks 2 and 3 are divided to obtain the ionospheric part; then, frequency shifts of uplink 1 and downlink 2 are divided. In step two, we substitute this result with the calculated residual Doppler effect and ionospheric part, so the gravitational potential difference can be calculated.

\subsection{Doppler residual errors}

For Doppler residuals in Sect. 3.1, we only consider Doppler shift difference between link 1 and link 2 while ignoring that of link 2 and link 3 . Since the time difference of link 2 and link 3 is less than $1 \mu \mathrm{s}\left(T_{34}<1 \mu \mathrm{s}\right)$, and they are both downlinks, Doppler shift difference between link 2 and link 3 is much smaller. Supposing that $T_{34}$ is $100 \mathrm{~ns}$, we take similar notes as Sect. 3.1 and Appendix B: A denotes time $t_{3}$, B denotes time $t_{5}, B^{\prime \prime}$ denotes time $t_{4}$, and $A^{\prime \prime}$ denotes time $t_{6}$. Based on the spatial relation, we have

$T_{56}=T_{34}-\frac{v_{\mathrm{A}} \cdot R_{\mathrm{AB}}}{c^{2}}+\frac{v_{\mathrm{B}} \cdot R_{\mathrm{AB}}}{c^{2}}$.

With a numerical calculation with Eq. (28), we find that the numerical difference between $T_{34}$ and $T_{56}$ is tens of nanosecond, which implies that both $T_{34}$ and $T_{56}$ are in the order of magnitude of $c^{-2}$ and must be corrected. Calculations show the Doppler shift difference between link 2 and link 3

$$
\begin{aligned}
& \frac{1-\frac{\mathbf{N}_{\mathrm{A}^{\prime \prime} \mathrm{B}^{\prime \prime}} \cdot \mathbf{v}_{\mathrm{B}^{\prime \prime}}}{c}}{1-\frac{\mathbf{N}_{\mathrm{A}^{\prime \prime} \mathrm{B}^{\prime \prime}} \cdot \mathbf{v}_{\mathrm{A}^{\prime \prime}}}{c}}-\frac{1-\frac{\mathbf{N}_{\mathrm{AB}} \cdot \mathbf{v}_{\mathrm{B}}}{c}}{1-\frac{\mathbf{N}_{\mathrm{AB}} \cdot \mathbf{v}_{\mathrm{A}}}{c}}=\frac{\left(\mathbf{N}_{\mathrm{AB}} \cdot \mathbf{v}_{\mathrm{B}}\right)}{c^{3}} \\
& {\left[\mathbf{N}_{\mathrm{AB}} \cdot\left(\mathbf{v}_{\mathrm{B}}-\mathbf{v}_{\mathrm{A}}\right)\right]^{2}-\frac{\left(\mathbf{v}_{\mathrm{B}}-\mathbf{v}_{\mathrm{A}}\right) \cdot \mathbf{v}_{\mathrm{B}} \mathbf{N}_{\mathrm{AB}} \cdot\left(\mathbf{v}_{\mathrm{B}}-\mathbf{v}_{\mathrm{A}}\right)}{c^{3}}} \\
& -\frac{\left(R_{\mathrm{AB}} \cdot a_{\mathrm{B}}\right) \mathbf{N}_{\mathrm{AB}} \cdot\left(\mathbf{v}_{\mathrm{B}}-\mathbf{v}_{\mathrm{A}}\right)}{c^{3}}+\left[\frac{\left[\mathbf{N}_{\mathrm{AB}} \cdot\left(\mathbf{v}_{\mathrm{B}}-\mathbf{v}_{\mathrm{A}}\right)\right]^{2}}{c R_{\mathrm{AB}}}\right. \\
& \left.-\frac{\left(\mathbf{v}_{\mathrm{B}}-\mathbf{v}_{\mathrm{A}}\right)^{2}}{c R_{\mathrm{AB}}}-\frac{\mathbf{N}_{\mathrm{AB}} \cdot\left(a_{\mathrm{B}}-a_{\mathrm{A}}\right)}{c}\right] T_{34} .
\end{aligned}
$$

Numerical calculations show that, $\left|\mathbf{N}_{\mathrm{AB}} \cdot \mathbf{v}_{\mathrm{A}} / c\right| \leq 2.6 \times$ $10^{-5},\left|\mathbf{N}_{\mathrm{AB}} \cdot \mathbf{v}_{\mathrm{B}} / c\right| \leq 1.6 \times 10^{-6},\left|\mathbf{R}_{\mathrm{AB}} \cdot \mathbf{a}_{\mathrm{A}} / c^{2}\right| \leq 1.7 \times$ $10^{-10},\left|\mathbf{R}_{\mathrm{AB}} \cdot \mathbf{a}_{\mathrm{B}} / c^{2}\right| \leq 7 \times 10^{-13}$, and the largest part in Eq. (29) is $\frac{\left[\mathbf{N}_{\mathrm{AB}} \cdot\left(\mathbf{v}_{\mathrm{B}}-\mathbf{v}_{\mathrm{A}}\right)\right]^{2}}{c R_{\mathrm{AB}}} T_{34}$, which will achieve $3 \times 10^{-14}$. Hence, assuming that $T_{34}=100 \mathrm{~ns}$, the maximal Doppler residual errors caused by link 2 and link 3 are $3 \times 10^{-14}$. Since 
$\left(\frac{f_{2}^{2}}{f_{1}^{2}}-1\right)\left(\frac{f_{3}^{2}}{f_{3}^{2}-f_{2}^{2}}\right)$ in Eq. (21) is approximately -0.0053 , this error will affect GRS Eq. (22) by a magnitude of $1.5 \times$ $10^{-16}$.

In Appendix B, we show the relevant approximations. We neglect the terms of $c^{-4}$, whose value are less than $10^{-17}$.

In summary, the maximal Doppler residual errors are $1.5 \times$ $10^{-16}$.

\subsection{Atmospheric residual errors}

In the expansion of the ionosphere refractive index, the terms of $f^{-3}$ cannot be eliminated by our TFC method and have not been considered in the former sections. According to the study of [36], the $f^{-3}$ terms of GNSS signals (L band) is approximately one in a hundred of $f^{-2}$ terms. By reckoning of our simulations, final effects caused by the $f^{-3}$ terms will be approximately $1 \times 10^{-15}$. Because of its variability and randomness, these errors will be effectively weakened to a considerably small value by averaging with a mass of data. The simulations in Sect. 5 will demonstrate that this effect can be lower than $10^{-16}$.

Moreover, there is a common residual error, that is, the atmospheric frequency shift will change with the location and time of links. Due to the location difference between link 1 and link 2 , the ground station moves about $1 \mathrm{~m}$ from $t_{1}$ to $t_{5}$. However, the spatial and temporal variations are very small, so it is difficult to analyze the difference of ionospheric and tropospheric frequency shifts. In general, the atmospheric composition changes on a large spatial scale, so this effect is ignored here.

\subsection{Relativistic residual errors}

The calculation of relativistic effects will also cause residuals. There are differences among relativistic effects of links 1, 2 and 3. However, in our analysis, we consider it a constant value. The relativistic differences between link 2 and link 3 are much smaller than those between link 1 and link 2 (the time difference is much smaller), so we will only consider the differences between link 1 and link 2. Relativistic effects include GRS effect and transverse Doppler effect. Regardless of ground displacements such as the solid Earth tide, the GRS effect only varies with the space station, but the transverse Doppler effect varies with both the space and ground station. Based on simulated data, the gravitational potential of space station varies by $100 \mathrm{~m}^{2} / \mathrm{s}^{2}$ per second. However, $T_{23}$ is at most $1 \mu \mathrm{s}$, so the GRS difference between link 1 and link 2 is approximately $10^{-20}$.

The transverse Doppler frequency shift is proportional to the square of velocity, and its differential expression is

$$
\left|d f_{\text {trans }}\right|=\frac{v}{c^{2}} d v=\frac{v a}{c^{2}} d t .
$$

Assuming that $T_{23}=1 \mu \mathrm{s}$ and $T_{15}=2 \mathrm{~ms}$, the maximal transverse Doppler frequency shift errors caused by the space and ground station are $7.6 \times 10^{-19}$ and $3 \times 10^{-19}$, respectively. Thus, the maximal transverse Doppler frequency shift error is around $1 \times 10^{-18}$.

\subsection{Tidal effects}

With regard to ground displacements, the relativistic effects have other residuals. According to IERS convention 2010 [27], displacements of reference points are divided into three categories: (1) Tidal motions (mostly near diurnal and semidiurnal frequencies) and other accurately modeled displacements of reference markers (mostly at longer periods); (2) other displacements of reference markers including nontidal motions associated with the changing environmental loads; (3) displacements that affect the internal reference points in the observing instruments.

We are interested in the first two types. Tidal motions include solid, ocean and polar tides. The solid tide has the largest magnitude, which will be tens of centimeters [27,37]. Other displacements include nontidal mass redistributions in the atmosphere, oceans, sea-level variations, etc., but their amplitude is much smaller (at most several centimeters) [38]. Crustal deformation and plate motions also contribute to the parameters $\left(\mathbf{r}_{\mathrm{B}}, \mathbf{v}_{\mathrm{B}}, \mathbf{a}_{\mathrm{B}}\right)$, which can be predicted by the tectonic model NUVEL-1 NNR of [39]. However, the numerical value of its kinematic vectors shows that the rate is only approximately several centimeters per year or hundreds of centimeters per day, which is 4 orders of magnitude smaller than the solid Earth tide ( $\sim$ tens of centimeters per day). Therefore, we will take the solid Earth tide as the dominant contribution.

Based on the IERS convention, we estimated the equatorial displacement, with the maximum value being $0.32 \mathrm{~m}$. If a sine function is used to estimate the change in displacement with time, we assume that all tides are diurnal tides, and the effect of the tides on the velocity is $4.65 \times 10^{-5} \mathrm{~m} / \mathrm{s}$. According to those estimates, the magnitude of the position and velocity vectors affects the residual Doppler terms in (22) and (23) by at most $6.76 \times 10^{-17}$, which is much less than the Doppler residuals analyzed in Sect. 3.1.

The solid Earth tide causes displacements and gravitational potential disturbance. The maximum tidal potentials caused by the moon and the sun are $4.41 \mathrm{~m}^{2} / \mathrm{s}^{2}$ and $1.60 \mathrm{~m}^{2} / \mathrm{s}^{2}$, respectively [40]. With regard to Love numbers $h, l$ and $k$, the gravitational potential tide will be $3.74 \mathrm{~m}^{2} / \mathrm{s}^{2}$, which is equivalent to a GRS of $4.2 \times 10^{-17}$, which is mainly caused by the semidiurnal tide.

Based on the above discussion in Sect. 4, the residual amount of each frequency shift is shown in Table 1. Among them, the Doppler frequency shift and ionosphere-related frequency shift are relatively large and can be manually elimi- 
Table 1 Relative magnitudes and residual amount of each frequency shift in the TFC method

\begin{tabular}{lll}
\hline Type & Magnitude & Residual \\
\hline Doppler frequency shift & $10^{-5} \sim 10^{-6}$ & $<1.5 \times 10^{-16}$ \\
Ionospheric frequency shift & $10^{-10} \sim 10^{-12} \leq 1 \times 10^{-15}$ \\
Tropospheric frequency shift & $10^{-13} \sim 10^{-14}<1 \times 10^{-18}$ \\
Gravitations red-shift (GRS) & $\leq 10^{-11}$ & - \\
Tidal effects on GRS & $4.2 \times 10^{-17}$ & - \\
Transverse Doppler frequency shift & $10^{-10} \sim 10^{-11}<1 \times 10^{-18}$ \\
Shapiro frequency shift & $\leq 10^{-14}$ & $<1 \times 10^{-18}$ \\
\hline
\end{tabular}

Table 2 Geographical parameters of Observatoire de Paris (OP)

Parameters Latitude Longitude Height Gravitational potential

Values $\quad 48.836^{\circ} \mathrm{N} \quad 2.336^{\circ} \mathrm{E} \quad 124.2 \mathrm{~m} \quad 62,573,855.538 \mathrm{~m}^{2} / \mathrm{s}^{2}$

nated if possible. The ionospheric part is difficult to manually eliminate, as demonstrated in further research (Sect. 5).

\section{Simulation experiments and results}

Since CSS is under construction, relevant parameters are not yet publically released. To test our theory and formulations, we present simulation experiments based on the onboarding ISS and the publically released information of ACES mission. Namely, we simulate the datasets of the ISS' real orbit, the ionosphere and troposphere models, the widely used gravity field model EGM2008 [41], the solid Earth tide model [40], and the simulated clock data by a conventionally accepted stochastic noises model $[42,43]$. Since the timefrequency system on board the CSS is similarly configured as ACES, our simulation results are also suitable for the CSS time-frequency system.

\subsection{Simulation setup and experiments}

In our simulations, we select the station Observatoire de Paris (OP) as the ground station with geographical parameters as shown in Table 2. In Sect. 3.3, we discussed that the magnitude of the tidal effect on GRS is approximately $10^{-17}$; nonetheless, in addition to the objective accuracy of $10^{-16}$, we still added the tidal effect to the simulation experiment. When tidal effects are neglected, the relevant parameters in ECEF related to the ground station OP are considered constant, as shown in Table 2. Other parameter settings in our experiment are listed in Table 3.

Figure 3 shows the procedures of the simulation experiments. The observations in our simulation experiments are carrier frequency values of ACES, which are denoted as $f_{1}^{\prime}$, $f_{2}^{\prime}$, and $f_{3}^{\prime}$, as described in Sect. 2. We can use a combination of $f_{1}^{\prime} / f_{1}, f_{2}^{\prime} / f_{2}, f_{3}^{\prime} / f_{3}$ (as Sect. 3 demonstrates) to calculate the gravitational potential difference between ISS and ground station and test the GRS.

To obtain the received frequency values $\left(f_{1}^{\prime}, f_{2}^{\prime}\right.$, and $\left.f_{3}^{\prime}\right)$, the original emitted frequency values and various types of frequency shifts are required: (1) Originally emitted frequency values of $f_{1}, f_{2}$, and $f_{3}$, and clock noises (including devices noises); (2) frequency shifts including Doppler frequency shift, relativistic frequency shift (including secondorder Doppler shift and GRS), atmospheric frequency shifts (including ionospheric and tropospheric parts), and tidal effects. To calculate these effects, we must have the position, velocity and acceleration information, which can be derived from the orbits of ISS and moving positions of the ground station. To select observable data, we set the condition that the observation elevation angle should be larger than $15^{\circ}$.

First, we must solve the emitted frequency values, which are frequency series composed of the given frequencies $f_{1}$, $f_{2}, f_{3}$ and clock noises. Based on the stochastic noise nature of the clocks, there are five types of clock noises: Random Walk FM (frequency modulation), Flicker FM, White FM, Flicker PM (phase modulation) and White PM [42] with spectral densities of the types $f^{-2}, f^{-1}, f^{0}, f^{1}$ and $f^{2}$, respectively.

Before starting our experiments, we provide samples of the simulated clock frequency noise series in Fig. 4. We simulated five pure types of noises with similar magnitudes (Fig. 4a-e) and their sum (Fig. 4f). The data length is 20,000. Figure $4 \mathrm{a}$ shows a strong trend, Fig. $4 \mathrm{~b}$ shows little trend and some periodic patterns, and Fig. $4 \mathrm{c}-\mathrm{e}$ show irregular patterns. White and random walk noises are the simple types [43]. Flicker noises were calculated by the AR (autoregressive) model [44].

In simulations, we took White FM as the dominant part because Allan deviation performance of PHARAO is very similar to that of pure white FM, and we simulated 864,000 s of clock data with sample intervals of $1 \mathrm{~s}$. The modified Allan deviation (MDEV, [42]) of our simulated data is shown in Fig. 5, and its performance is similar with previous studies $[13,15]$. Figure 5 shows a potential of long-term stability of $10^{-16}$. With these clock data, we succeeded in the first step: the emitted frequency values were generated.

In the experiment, to simulate the orbit, we use the daily orbit elements to calculate. The TEC (Total Electron Content) data are interpolated from the grid data, which are derived from CDDIS (The Crustal Dynamics Data Information System). Using the TEC data and time-varying rate, we can simulate the ionospheric frequency shift. The refractive frequency shift caused by the ionosphere is calculated by the refractive angle, which is integrated from the layered structure model [25] of ionosphere. The troposphere is calculated by the zenith tropospheric delay (ZTD) of the dry and wet 
Table 3 Relevant parameters in the simulation experiment

\begin{tabular}{llll}
\hline Parameter & Value & Parameter & Value \\
\hline Earth radius $R$ & $6,378,137 \mathrm{~m}$ & Threshold of observation elevation & $15^{\circ}$ \\
Earth flatness $e^{2}$ & 0.006694 & Light speed in vacuum $c$ & $299,792,458 \mathrm{~m} / \mathrm{s}$ \\
$T_{23}$ & $1 \times 10^{-6} \mathrm{~s}$ & Peak ionospheric height & $200 \mathrm{~km}$ \\
$T_{34}$ & $1 \times 10^{-7} \mathrm{~s}$ & Peak electron density & $3 \times 10^{12} \mathrm{~m}^{-3}$ \\
Gravitational constant $G M$ & $3.9860 \times 10^{14} \mathrm{~N} \mathrm{~m}^{2} / \mathrm{kg}$ & Doodson's constant & $26,277 \mathrm{~cm}^{2} / \mathrm{s}^{2}$ \\
\hline
\end{tabular}

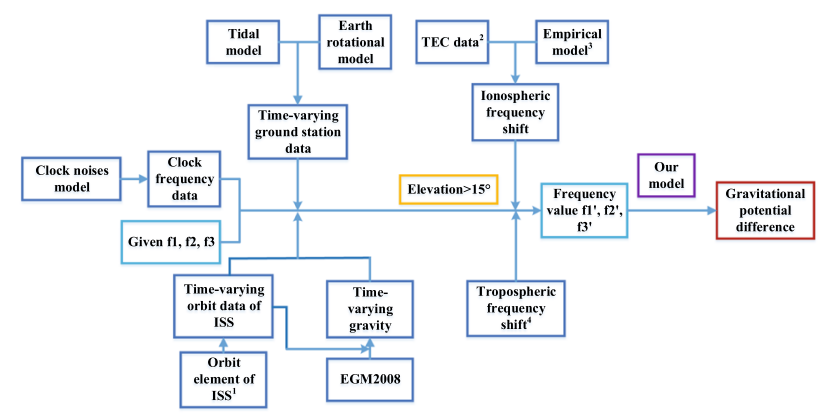

Fig. 3 Diagram of the simulation experiments. The superscripts in red in this figure indicate that: 1. six orbit elements obtained from http:// spaceflight.nasa.gov/; 2. TEC data obtained from ftp://cddis.gsfc.nasa. gov/; 3 . Model of the refractive index varying with height; 4 . Wet and dry ZTDs obtained from http://ggosatm.hg.tuwien.ac.at/
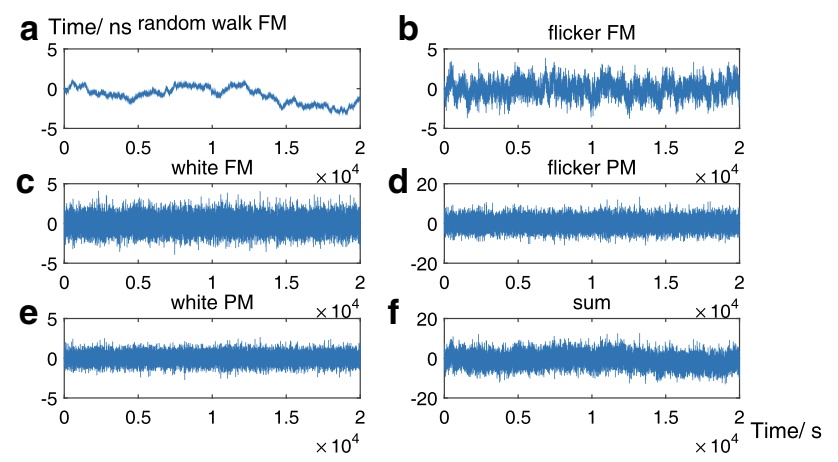

Fig. 4 Sample of simulated clock noises. a Random walk FM series; b Flicker FM series; c White FM series; d Flicker PM series; e White PM series; $\mathbf{f}$ Sum of those five types of noises. Units of the vertical and horizontal axes are ns and $10^{4} \mathrm{~s}$, respectively

components, and the projection function adopted the Vienna Mapping Function (VMF1) mode. The refractive frequency shift caused by the ionosphere is not calculated because this part is independent of the carrier frequency and easy to eliminate. We adopted EGM2008 [41] for gravitational potential models. We considered the effect of the tidal effect on the ground gravitational potential because this is the main source of residual error in GRS. For this part, we first calculated the Earth tide generated potential from parameters of periodic tides [40] using the method of harmonic analysis. Then, we considered Love numbers and solved the gravitational potential tide. Combining with the analysis in Sect. 4, we did not

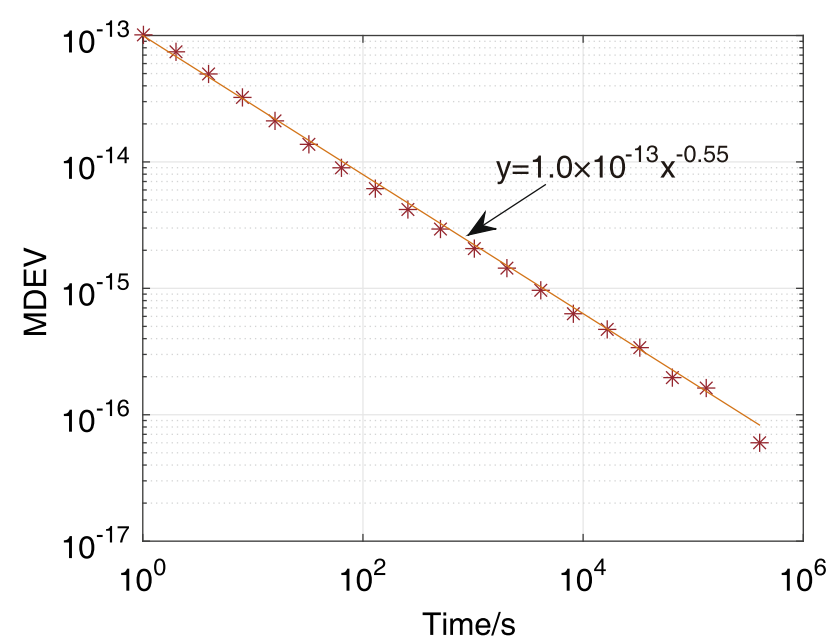

Fig. 5 Modified Allan Deviation (MDEV) of the simulated clock errors. The points refer to the calculated MDEV data, and the line is fitted from the points

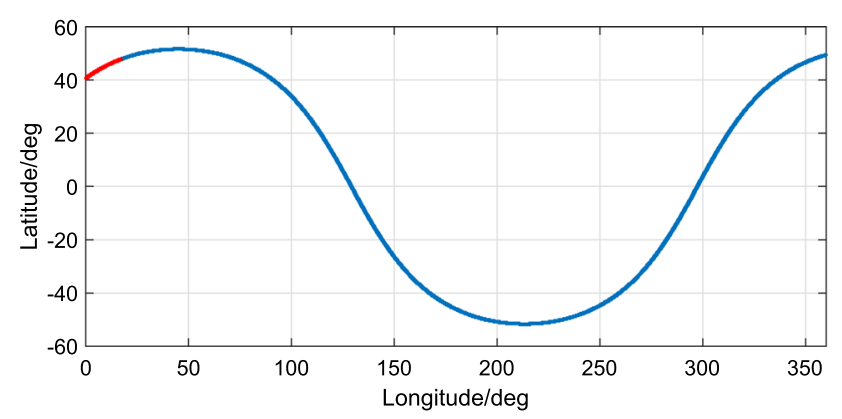

Fig. 6 ISS's track of the subsatellite point. The red part of the curve denotes the position of ISS that can be directly observed, and the blue part of the curve denotes the trajectory of an entire circle

consider the indirect tidal effect on the coordinates variations of the ground station because this indirect tide is much smaller than other residual errors.

ISS is flying in an orbit with an inclination of $51.6^{\circ}$ and a period of $5400 \mathrm{~s}$, and its track of the subsatellite point is shown in Fig. 6. In this figure, the red part is where we can observe. Because ISS is flying in a low orbit with a large velocity, each pass over the ground station will last approximately $600 \mathrm{~s}$ at most [17]; for our elevation threshold of $15^{\circ}$, we can only observe approximately 300 s per pass. 


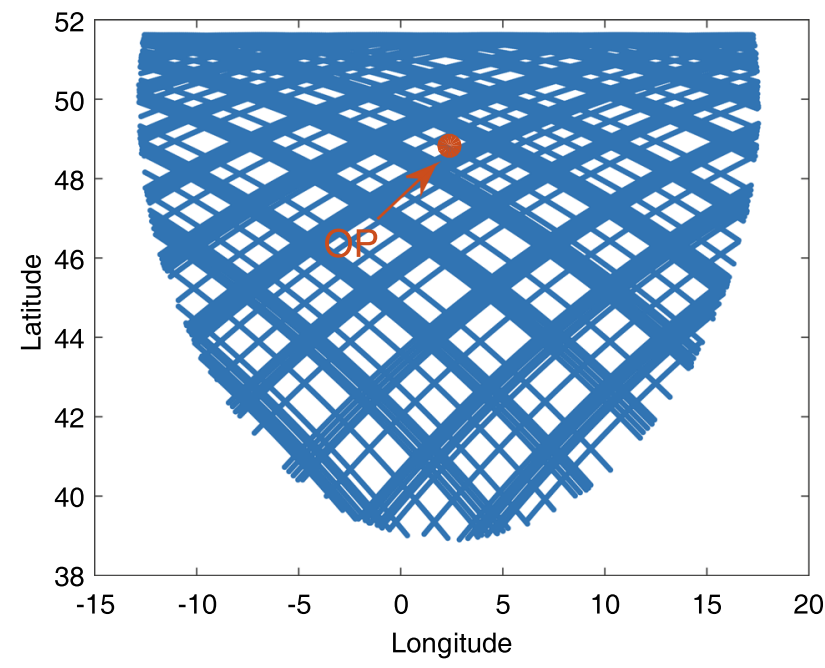

Fig. 7 Track of the subsatellite point of all passes of ISS in 29 days of observation

\subsection{Results and accuracy level of the test}

For our experiment, we had 29-day simulated observations, and ISS flew above OP 130 times in total. As shown in Fig. 7, we drew a subsatellite point trajectory with the station OP at the center. Because the inclination of ISS is $51.6^{\circ}$, the maximal latitude of the subsatellite point is that value.

After analyzing the orbits of ISS, we selected the time when we could observe with elevations greater than $15^{\circ}$. Based on the position information of ISS and OP, downloaded atmospheric parameters, gravitational quantities and various models, we calculated all types of frequency shifts as shown in Fig. 3, whose magnitudes are shown in Table 1. The result shows that Doppler frequency shift is the dominant part, while Shapiro frequency shift is the smallest part. Refraction effects cannot be neglected because they have larger magnitude than the ionospheric frequency shift and tropospheric frequency shift. In the experiment, although the residuals of some errors (the higher-order term of the ionosphere and the Doppler residual errors) are greater than $10^{-16}$, due to the randomness of the errors, simulation experiments show that after a long-term average, they can be less than $10^{-16}$.

Furthermore, we analyzed each frequency shift in a oneway transfer (link 3, Fig. 8) and the residual of various frequency shifts after applying the TFC method (Fig. 9). For the time length, we only show one pass. Figure 8 is consistent with Table 1, where the relativistic frequency shift appears unchanged because the gravitational potential and velocity norm of ISS slowly change with time. There appears to be singularities in Fig. 8, but those are not real. When ISS was exactly on zenith of the station, the velocity became vertical to the line of sight (LoS), which made Doppler and Shapiro effects extremely small and resemble a singularity.

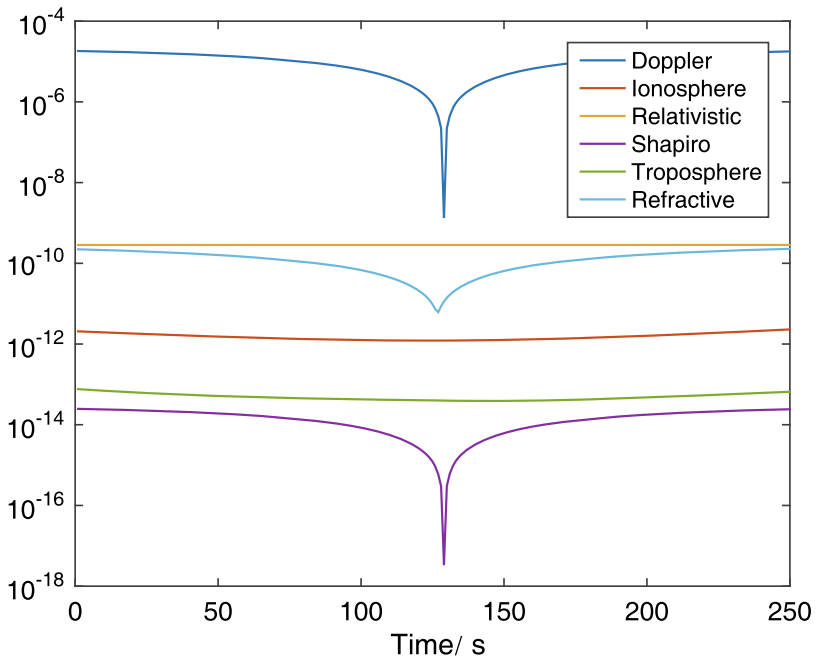

Fig. 8 Different colored curves denote different relative frequency shifts in a one-way transfer

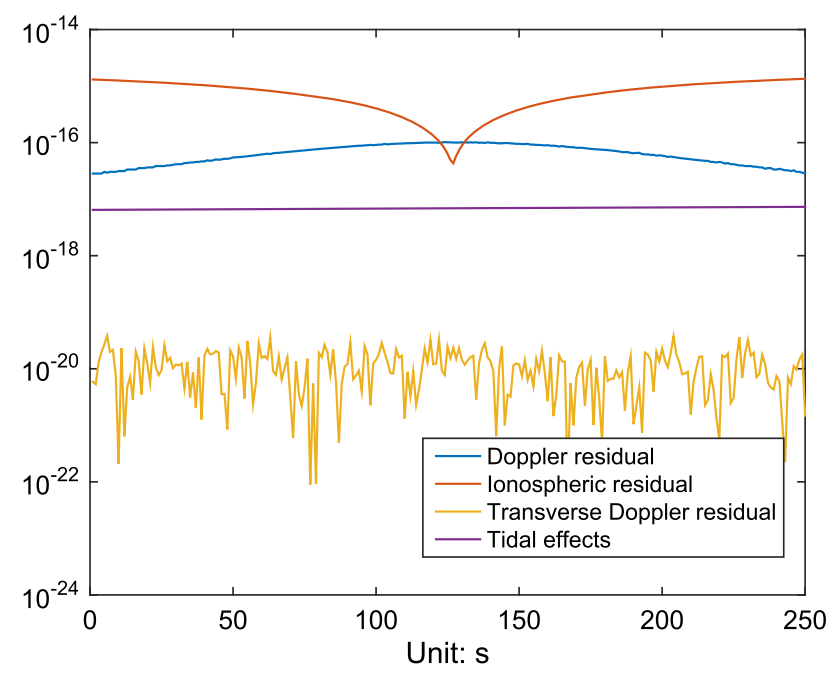

Fig. 9 Residual errors of various frequency shifts

The TFC method can largely eliminate various errors, but the analysis in Sect. 4 shows that residual errors remain. According to Table 1, we calculated the Doppler residuals, ionospheric-related residuals (including ionospheric frequency shift and ionospheric refraction), transverse Doppler residuals, and tidal effects. The Doppler residual is caused by the Doppler difference between link 2 and link 3 . The ionospheric-related residual is caused by the higher-order ionospheric term. The transverse Doppler residual is caused by the change in velocity. Figure 9 shows that among the residuals of various relative frequency shifts, the largest component is the ionospheric residual, which can reach a maximum of $1 \times 10^{-15}$ and is often at the level of $10^{-16}$; the second largest component is the Doppler residual, whose maximum is $1 \times 10^{-16}$; the magnitudes of other frequency shifts are much smaller than $10^{-16}$, so they are negligible. 


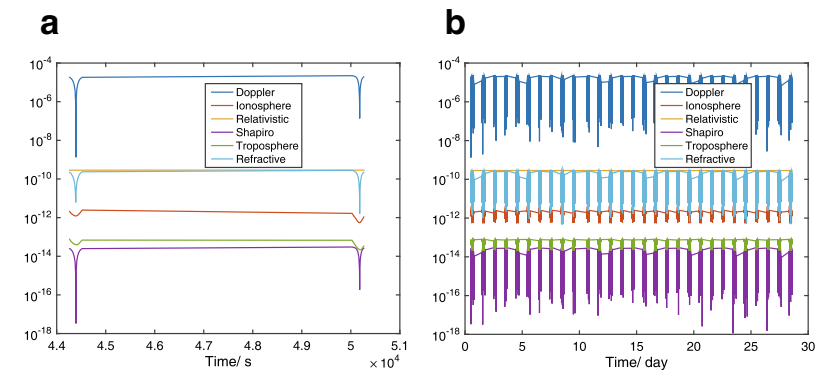

Fig. 10 a Various frequency shifts in a one-way link of two adjacent epochs; b various frequency shifts in a one-way link in the 29-day observation

These two frequency shifts should be corrected in the TFC method model, but simulation experiments show that due to the variability of these two frequency shifts, they can be eliminated below $10^{-16}$ after a long-term average, so they can be ignored. Thus, the TFC method model can indeed eliminate the largest Doppler frequency shift. The magnitudes of various residual errors are consistent with the estimates in Table 1.

In addition to the discussed systematic residual errors, random errors will be caused by the parameters of the ground station and space station in the results. We will later analyze the impact of these errors.

Figures 8 and 9 only show the fractional frequency shift during a single flight of the space station, and Fig. 10 shows the data for a longer time period. We define the observations of one single pass of ISS as an epoch. Figure 10a shows 2 closed epochs, and Fig. 10b shows the entire data (130 epochs). Time intervals between two epochs are very large and much larger than the time duration of one epoch itself. Thus, we can solve these data epoch by epoch, obtain the averaged results, and evaluate the results of each epoch.

Finally, we obtained the received frequency values $\left(f_{1}^{\prime}\right.$, $f_{2}^{\prime}$ and $f_{3}^{\prime}$ ). Using our TFC model as Sect. 3 proposed, we can obtain the gravitational potential differences (blue line) and compared them with the real differences calculated by EGM2008 in Fig. 11a. The results are obtained by only one single epoch, and the dotted line in the figure is the range of the error in the $1-\sigma$ criterion. Figure $11 \mathrm{~b}$ shows the gravitational potential differences after averaging epoch by epoch. The results show that the precision is not good for data sampled per second (Fig. 11a), which is equivalent to $250 \mathrm{~m}$ in height, which is within our expectation. However, after the epoch-by-epoch averaging, we obtained a gravitational potential (GP) difference bias series. The precision of the gravitational potential differences has improved to approximately $218 \mathrm{~m}^{2} / \mathrm{s}^{2}$ (approximately equivalent to an elevation of $22.2 \mathrm{~m}$ ), as shown in Fig. 11b. Since the slope on the MDEV plot of Fig. 5 is around $-1 / 2$, our simulated noise is approximately nor-
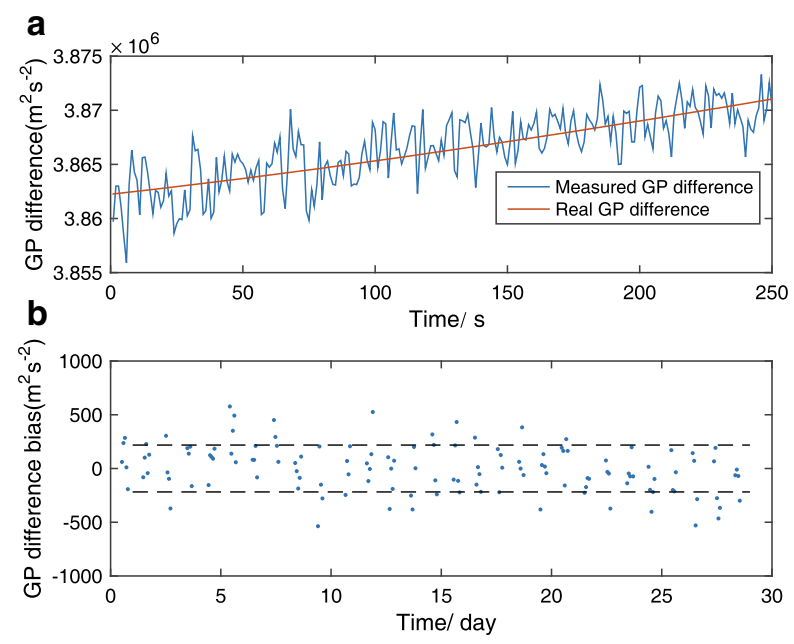

Fig. 11 a Various frequency shifts in a one-way link of two adjacent epochs; b various frequency shifts in a one-way link in the 29-day observation

Table 4 Gravitational potential difference and accuracy of test

\begin{tabular}{lll}
\hline Parameters & Value & Uncertainty \\
\hline Model & $3,834,161.3 \mathrm{~m}^{2} / \mathrm{s}^{2}$ & $3 \mathrm{~m}^{2} / \mathrm{s}^{2}$ \\
Observation & $3,834,165.3 \mathrm{~m}^{2} / \mathrm{s}^{2}$ & $18.6 \mathrm{~m}^{2} / \mathrm{s}^{2}$ \\
Relative accuracy & $1.04 \times 10^{-6}$ & $4.91 \times 10^{-6}$ \\
\hline
\end{tabular}

mally distributed [42]. Thus we applied standard Gaussian statistics to evaluate the uncertainty. Results are shown in Table 4. After averaging the entire data set, we obtain a gravitational potential difference bias of $4.0 \pm 18.6 \mathrm{~m}^{2} / \mathrm{s}^{2}$. Compared with the real averaged gravitational potential difference of $3.8340 \times 10^{6} \mathrm{~m}^{2} / \mathrm{s}^{2}$, our testing precision achieves $(1.04 \pm 4.85) \times 10^{-6}$. Supposing that the gravitational potential difference calculated by EGM2008 has an uncertainty of $3 \mathrm{~m}^{2} / \mathrm{s}^{2}$, the testing uncertainty achieves $\sqrt{18.6^{2}+3^{2}} /\left(3.8340 \times 10^{6}\right) \approx 4.91 \times 10^{-6}$; therefore, our testing precision is $(1.04 \pm 4.91) \times 10^{-6}$. Here, we only used 29 days of observations. With longer-period experiments, the results will be better, considering the noise characteristics.

\subsection{Accuracy requirements of relevant parameters}

For the calculation of our TFC model, we examine the requirements of the parameter precision (Table 5). To obtain results with high precision, we need parameters to satisfy these demands. $\mathbf{r}_{\mathrm{A}}$ and $\mathbf{r}_{\mathrm{B}}$ are the positions of ISS and the ground station, respectively; $\mathbf{v}_{\mathrm{A}}$ and $\mathbf{v}_{\mathrm{B}}$ are their velocities; $\mathbf{a}_{\mathrm{A}}$ and $\mathbf{a}_{\mathrm{B}}$ are their accelerations; $T_{23}$ is the time offset of link 1 and link 2. All ground station parameters here can be accurately obtained based on the coordinates and Earth rotation model and do not need to be discussed in detail. Thus, we must only study the parameters related to the space station. 
Table 5 Minimal required accuracy of parameters calculated based on simulation data; in the case, the accuracy requirement for GRS is $1 \times 10^{-16}$

\begin{tabular}{llll}
\hline Parameters & Demand along track & Horizontal demand & Radial demand \\
\hline $\mathbf{r}_{\mathrm{A}}$ & $240 \mathrm{~m}$ & $689 \mathrm{~m}$ & $465 \mathrm{~m}$ \\
$\mathbf{v}_{\mathrm{A}}$ & $1.23 \mathrm{~m} / \mathrm{s}$ & $3.26 \mathrm{~m} / \mathrm{s}$ & $2.48 \mathrm{~m} / \mathrm{s}$ \\
$\mathbf{a}_{\mathrm{A}}$ & $69.6 \mathrm{~m} / \mathrm{s}^{2}$ & $80.0 \mathrm{~m} / \mathrm{s}^{2}$ & $65.3 \mathrm{~m} / \mathrm{s}^{2}$ \\
$T_{23}$ & & $5.5 \times 10^{-7} \mathrm{~s}$ & \\
\hline
\end{tabular}

These parameters are demanded for the calculation of Eqs. (22) and (23); thus, we derived the total differential of these equations and analyzed the differential relationship between parameters $\left(\mathbf{r}_{\mathrm{A}}, \mathbf{v}_{\mathrm{A}}, \mathbf{a}_{\mathrm{A}}\right.$ and $\left.T_{23}\right)$ and gravitational potential difference. The measurement noises are stochastic, so after averaging, the noises will be largely weakened. Due to this principle, based on the MDEV curve of ACES clocks, we set a target precision of $10^{-13}$ at a sampling rate of 1 second.

According to Table 5, the requirements of orbit parameters and acceleration parameters are easy to satisfy, and $T_{23}$ is easy to control at that level. Only the precision requirement of the velocity is high and must be carefully solved. With space technology nowadays, it is easy for a to satisfy these demands. In our parameter analysis, for each analysis of one parameter, we suppose that the other parameters are constant. In this case, we may clearly examine the effect of the error of each parameter on the results. In the real environment, there are stochastic errors in all parameters. Table 5 shows that only the velocity is the principal error source if all errors exist. Therefore, our parameter analysis scheme is feasible.

\section{Conclusion}

In this study, we proposed a one-way frequency transfer model based on the frequency transfer to order $c^{-3}$ [23] and Doppler shift considering the refractive effect [31]. This model is established in free space and has an accuracy of $c^{-3}$. Then, we proposed the TFC method to derive the GRS based on three frequency links. Different from other combination methods addressed to ACES [17,21] that use time comparison, our model addresses the frequency comparison, which will be available on CSS mission. This combination method can largely eliminate Doppler frequency shift, atmospheric effects, etc. We also estimated magnitudes of the residual errors and provided models to eliminate them. Furthermore, we designed simulation experiments to authenticate our model and analyzed the demanded parameters. The simulation results show that the proposed model in this study can achieve a relative accuracy level of $\beta=(1.04 \pm 4.91) \times 10^{-6}$.

Equipped with atomic clocks with a frequency stability of $(1-3) \times 10^{-16}$, our study shows that the test of GRS can achieve better performance if longer data (say much longer data than 29 days) are obtained due to the noise characteristics, which is one and a half orders higher than the accuracy level given by [9]. Comparing with the formerly proposed time comparison, our method is not relevant with phase ambiguity, can determine the gravitational potential within a short time interval, and simulation results show a less requirement of averaging time. This study provides a new approach to test the GRS and benefits the brand-new field of relativistic geodesy.

Due to the limitation of the accuracy of the atomic clock it carries, the testing precision is at the $10^{-6}$ level. To achieve higher scientific goals, more precise space atomic clocks and time-frequency comparison links are required. The CSS provides this opportunity. Since the CSS will carry an opticatomic clock at the frequency stability level of $8 \times 10^{-18} /$ day $[19,20]$, which is at least one order of magnitude higher than the cold atomic cesium clock that ACES carries. It is expected to achieve $10-\mathrm{cm}$ to $5-\mathrm{cm}$ level in equivalent height to the gravitational potential measurements and can test the GRS at a level of $10^{-7}$.

However, due to the higher accuracy of the clock on the CSS, there are differences in model and actual measurement. In terms of the model, it must consider the time-frequency comparison model under the relativistic framework of $c^{-4}$ accuracy, and some of the originally negligible residual items in Sect. 4 must be carefully considered. In addition, because the magnitude of the high-order ionospheric term is obvious and difficult to eliminate, one may consider a potential solution to generalize the TFC method to a four-frequency combination and remove the high-order ionospheric terms. If the hardware conditions cannot satisfy the requirements of the quad-band link, a more in-depth study of the ionospheric frequency shift is required. If the accuracy of the model must reach the level of $10^{-18}$, it is also necessary to consider the tidal effect.

In terms of actual measurement, due to the higher accuracy of gravitational potential, according to the model, there are higher-accuracy requirements in measuring the position and speed of the space station, which introduces further requirements on the positioning system of the space station and hardware facilities for time measurement. If the accuracy requirement is increased by one and a half orders of magnitude, the velocity of space station should achieve a higher accuracy 
level. In summary, the formulation of the TFC method proposed in this study is also feasible for the CSS plan.

Acknowledgements We would like to express our sincere thanks to an anonymous Reviewer and A. Lindner for their valuable comments and suggestions, which greatly improved the manuscript. This study is supported by National Natural Science Foundation of China (Grant nos. 41631072, 42030105, 41721003, 41804012, 41874023, 41974034), Space Station Project (2020)228 and Natural Science Foundation of Hubei Province (grant No. 2019CFB611).

Data Availability Statement This manuscript has no associated data or the data will not be deposited. [Authors' comment: The simulation datasets used in this study are available on reasonable request from the corresponding author.]

Open Access This article is licensed under a Creative Commons Attribution 4.0 International License, which permits use, sharing, adaptation, distribution and reproduction in any medium or format, as long as you give appropriate credit to the original author(s) and the source, provide a link to the Creative Commons licence, and indicate if changes were made. The images or other third party material in this article are included in the article's Creative Commons licence, unless indicated otherwise in a credit line to the material. If material is not included in the article's Creative Commons licence and your intended use is not permitted by statutory regulation or exceeds the permitted use, you will need to obtain permission directly from the copyright holder. To view a copy of this licence, visit http://creativecomm ons.org/licenses/by/4.0/.

Funded by SCOAP ${ }^{3}$.

\section{Appendix A}

In our modeling, we focus on the down link from A to B, but since the optical path is reversible, we draw Fig. 1 as if it is the link from B to A. Assuming that our Earth is a sphere, and the atmosphere is composed of many thin spherical shells, in each of which the medium is homogeneous. Based on Bouguer's law (Snell's law in spherical surface) $[36,45]$, the following equation holds

$a=n r \sin i=\mathrm{const}$

where $n$ is the refractive index, and $i$ is the zenith angle at each layer.

For electromagnetic waves that propagate in a medium with refractive index $n$, the differential bending angle $d \boldsymbol{\alpha}$ that accrue on the ray path over a differential arc length $d s$ is given by [33]

$d \boldsymbol{\alpha}=n^{-1}(\mathbf{T} \times \nabla n) d s$

where $\mathbf{T}$ is its unit tangent vector defined by $\mathbf{T}=\mathbf{k} / k, \mathbf{k}(r)$ is the vector wavenumber of the ray at point $r$, and $\Delta n$ is the gradient vector of the refraction index. Here, differential bending angle $d \boldsymbol{\alpha}$ is written in vector form and related to the value and direction.
The refraction index in the ionosphere is displayed by Eq. (8)

$n=1-40.3 \frac{n_{\mathrm{e}}}{f^{2}}$.

We suppose that the refractive index only changes in the vertical direction. At the lower middle part of the ionosphere layer, electron density $n_{\mathrm{e}}$ is at the peak [25]. If we integrate Eq. (A.2) from the peak height to the space station height, the gradient of the refractive index can be expressed as a vertical downward vector form, and the integration is

$\boldsymbol{\alpha}=\int_{P} n^{-1}(\mathbf{T} \times \nabla n) d s=\int_{\mathrm{h}_{\max }}^{h_{\mathrm{SS}}} n^{-1} \frac{d n}{d h} \frac{\sin i}{\cos i} \mathbf{e}_{\mathrm{g}} d h$

where $\mathbf{e}_{\mathrm{g}}$ is the unit vector whose direction is defined by $\mathbf{T} \times \nabla n$. According to Eq. (A.3), $\partial n / \partial h$ is a positive value, and we use

$$
\begin{aligned}
\alpha & =\int_{0}^{h_{\mathrm{SS}}} n^{-1} \frac{d n}{d h} \frac{\sin i}{\cos i} d h=\int_{0}^{h_{\mathrm{SS}}} n^{-1} \frac{d n}{d h} \frac{\frac{a}{n\left(h+R_{\mathrm{E}}\right)}}{\sqrt{1-\left(\frac{a}{n\left(h+R_{\mathrm{E}}\right)}\right)^{2}}} d h \\
& =\int_{0}^{h_{\mathrm{SS}}} n^{-1} \frac{d n}{d h} \frac{a}{\sqrt{n^{2}\left(h+R_{\mathrm{E}}\right)^{2}-a^{2}}} d h
\end{aligned}
$$

where $R_{\mathrm{E}}$ is the Earth's average radius.

For the links between a ground station and a space station, we consider the ionospheric part (discussed in Sect. 2), and we let [25]

$n_{\mathrm{e}}(h)= \begin{cases}N_{\max } \exp \left(-\frac{h-h_{\max }}{H}\right) & h>h_{\max } \\ 0 & \text { otherwise }\end{cases}$

where $h_{\max }$ and $N_{\max }$ correspond to the peak height and peak density, respectively; $H$ is the free electron density scale height. Considering (A.4) and (A.5), we have the following for the ionosphere

$\frac{d n}{d h}=\frac{40.3 N_{\max }}{H f^{2}} \exp \left(-\frac{h-h_{\max }}{H}\right)$.

Substituting (A.7) into (A.5) and taking $n=1$ for approximation, Eq. (A.5) gives

$\alpha_{\text {ion }}=\frac{40.3 a N_{\max }}{H f^{2}} I$

where integration $I$ is

$$
I=\int_{h_{\max }}^{h_{\mathrm{SS}}} \frac{1}{\sqrt{n^{2}\left(h+R_{\mathrm{E}}\right)^{2}-a^{2}}} \exp \left(-\frac{h-h_{\max }}{H}\right) d h .
$$


It is weekly correlated to carrier frequency $f$, and the tropospheric part can be determined in this manner. For the total bending angle, we have

$\alpha=\alpha_{\text {ion }}+\alpha_{\text {trop }}$

Based on the reversible principle of the optical path, for three frequency signals, the only difference is carrier frequency $f$. Moreover, we have $\alpha_{\text {ion }} \sim 1 / f^{2}$.

To calculate the effect of refraction on the Doppler effect, we must separately calculate the deflection angles at $\mathrm{A}$ and B. If we define $\delta_{\mathrm{A}}$ and $\delta_{\mathrm{B}}$ as the deflection angles between the direction of the ray and the direct connection of $\mathrm{A}$ and $\mathrm{B}$, which implies that

$\alpha=\delta_{\mathrm{A}}+\delta_{\mathrm{B}}$

As Fig. 1 shows, we have

$\begin{aligned} \delta_{\mathrm{A}} & =\beta_{\mathrm{A}}-\theta_{\mathrm{A}} \\ \delta_{\mathrm{B}} & =\theta_{\mathrm{B}}-\beta_{\mathrm{B}} .\end{aligned}$

According to Eqs. (A.1) and (A.12), we have

$n_{\mathrm{A}} r_{\mathrm{A}}\left(\sin \theta_{\mathrm{A}}+\delta_{\mathrm{A}} \cos \theta_{\mathrm{A}}\right)=n_{\mathrm{B}} r_{\mathrm{B}}\left(\sin \theta_{\mathrm{B}}-\delta_{\mathrm{B}} \cos \theta_{\mathrm{B}}\right)$.

For Eqs. (A.10), (A.11) and (A.13), $\delta_{\mathrm{A}}$ and $\delta_{\mathrm{B}}$ can be solved by the expression of $\alpha$

$\begin{aligned} \delta_{\mathrm{A}} & =-\frac{n_{\mathrm{A}} r_{\mathrm{A}} \sin \theta_{\mathrm{A}}-n_{\mathrm{B}} r_{\mathrm{B}} \sin \theta_{\mathrm{B}}+\alpha n_{\mathrm{B}} r_{\mathrm{B}} \cos \theta_{\mathrm{B}}}{n_{\mathrm{A}} r_{\mathrm{A}} \cos \theta_{\mathrm{A}}-n_{\mathrm{B}} r_{\mathrm{B}} \cos \theta_{\mathrm{B}}} \\ \delta_{\mathrm{B}} & =\frac{n_{\mathrm{A}} r_{\mathrm{A}} \sin \theta_{\mathrm{A}}-n_{\mathrm{B}} r_{\mathrm{B}} \sin \theta_{\mathrm{B}}+\alpha n_{\mathrm{A}} r_{\mathrm{A}} \cos \theta_{\mathrm{A}}}{n_{\mathrm{A}} r_{\mathrm{A}} \cos \theta_{\mathrm{A}}-n_{\mathrm{B}} r_{\mathrm{B}} \cos \theta_{\mathrm{B}}}\end{aligned}$

From Eq. (A.14), $n_{\mathrm{A}}$ and $\alpha$ are relevant with carrier frequency $f$ (the space station is in the ionosphere layer, the term $n_{\mathrm{A}}-1$ is proportional to $f^{-2}$, and the ground station is in the troposphere layer), and the term $n_{\mathrm{A}} r_{\mathrm{A}} \cos \theta_{\mathrm{A}}-n_{\mathrm{B}} r_{\mathrm{B}} \cos \theta_{\mathrm{B}}$ is nearly irrelevant to carrier frequency $f$ because the term $n_{\mathrm{A}}-1$ is small compared to the entire term. Thus, Eq. (A.14) can be divided into two parts: the first part is irrelevant to carrier frequency $f$, and the second part is proportional to $f^{-2}$.

$$
\begin{aligned}
\delta_{\mathrm{A}}= & \delta_{\mathrm{A}}^{0}+\delta_{\mathrm{A}}^{\text {ion }} \\
= & -\frac{r_{\mathrm{A}} \sin \theta_{\mathrm{A}}-n_{\mathrm{B}} r_{\mathrm{B}} \sin \theta_{\mathrm{B}}+\alpha_{\text {trop }} n_{\mathrm{B}} r_{\mathrm{B}} \cos \theta_{\mathrm{B}}}{n_{\mathrm{A}} r_{\mathrm{A}} \cos \theta_{\mathrm{A}}-n_{\mathrm{B}} r_{\mathrm{B}} \cos \theta_{\mathrm{B}}} \\
& -\frac{\left(n_{\mathrm{A}}-1\right) r_{\mathrm{A}} \sin \theta_{\mathrm{A}}+\alpha_{\text {ion }} n_{\mathrm{B}} r_{\mathrm{B}} \cos \theta_{\mathrm{B}}}{n_{\mathrm{A}} r_{\mathrm{A}} \cos \theta_{\mathrm{A}}-n_{\mathrm{B}} r_{\mathrm{B}} \cos \theta_{\mathrm{B}}} \\
\delta_{\mathrm{B}}= & \delta_{\mathrm{B}}^{0}+\delta_{\mathrm{B}}^{\text {ion }} \\
= & \frac{r_{\mathrm{A}} \sin \theta_{\mathrm{A}}-n_{\mathrm{B}} r_{\mathrm{B}} \sin \theta_{\mathrm{B}}+\alpha_{\text {trop }} n_{\mathrm{A}} r_{\mathrm{A}} \cos \theta_{\mathrm{A}}}{n_{\mathrm{A}} r_{\mathrm{A}} \cos \theta_{\mathrm{A}}-n_{\mathrm{B}} r_{\mathrm{B}} \cos \theta_{\mathrm{B}}} \\
& +\frac{\left(n_{\mathrm{A}}-1\right) r_{\mathrm{A}} \sin \theta_{\mathrm{A}}+\alpha_{\text {ion }} n_{\mathrm{A}} r_{\mathrm{A}} \cos \theta_{\mathrm{A}}}{n_{\mathrm{A}} r_{\mathrm{A}} \cos \theta_{\mathrm{A}}-n_{\mathrm{B}} r_{\mathrm{B}} \cos \theta_{\mathrm{B}}} .
\end{aligned}
$$

To calculate $\mathbf{T} \cdot \mathbf{v}$ in Eq. (14), we must project these two vectors to the same plane. We set point $B$ to be the origin, direction $\mathrm{OB}$ to be the $\mathrm{y}$-axis, and the tangent of the Earth surface to be the $\mathrm{x}$-axis, and we obey the right-hand rule to define the $\mathrm{z}$-axis. This coordinate system is defined as the Local Link Coordinates System (LLCS). We can transform vectors from ECEF to LLCS and select the $\mathrm{x}$ and y components in LLCS, which are the projected coordinates. In the xoy plane of LLCS, vectors $\mathbf{T}_{\mathrm{A}}$ and $\mathbf{T}_{\mathrm{B}}$ can be expressed as

$\mathbf{T}_{\mathrm{A}}=\left[\cos \left(\gamma_{\mathrm{B}}-\delta_{\mathrm{A}}\right), \sin \left(\gamma_{\mathrm{B}}-\delta_{\mathrm{A}}\right)\right]$

$\mathbf{T}_{\mathrm{B}}=\left[\cos \left(\gamma_{\mathrm{B}}+\delta_{\mathrm{B}}\right), \sin \left(\gamma_{\mathrm{B}}+\delta_{\mathrm{B}}\right)\right]$.

The velocity vectors (of both the space and ground station) can be transformed by

$$
\begin{aligned}
& \mathbf{v}_{\text {LLCS }}=A^{-1} \mathbf{v} \\
& A=\left[\begin{array}{lll}
\mathbf{e}_{x} & \mathbf{e}_{y} & \mathbf{e}_{z}
\end{array}\right] .
\end{aligned}
$$

Here, $A$ is the transition matrix, and $\mathbf{e}_{x}, \mathbf{e}_{y}$, and $\mathbf{e}_{z}$ can be solved by

$\mathbf{e}_{x}=\frac{\mathbf{n}_{x}\left|\mathbf{n}_{x} \cdot \mathbf{r}_{\mathrm{OA}}\right|}{\mathbf{n}_{x} \cdot \mathbf{r}_{\mathrm{OA}}\left|\mathbf{n}_{x}\right|}$

$\mathbf{e}_{y}=\frac{\mathbf{r}_{\mathrm{OB}}}{\left|\mathbf{r}_{\mathrm{OB}}\right|}$

$\mathbf{e}_{z}=\mathbf{e}_{x} \cdot \mathbf{e}_{y}$

$\mathbf{n}_{x}=\mathbf{r}_{\mathrm{OA}}-\frac{\mathbf{r}_{\mathrm{OA}} \cdot \mathbf{r}_{\mathrm{OB}}}{\left|\mathbf{r}_{\mathrm{OB}}\right|^{2}} \mathbf{r}_{\mathrm{OB}}$

where $\mathbf{r}_{\mathrm{OA}}$ and $\mathbf{r}_{\mathrm{OB}}$ are vectors of $\mathrm{OA}$ and $\mathrm{OB}$ in ECEF.

We select the $\mathrm{x}$ and $\mathrm{y}$ components of $\mathbf{v}_{\mathrm{A}}$ and $\mathbf{v}_{\mathrm{B}}$ after coordinate transferring to be $v_{\mathrm{Ax}}, v_{\mathrm{Ay}}, v_{\mathrm{Bx}}$ and $v_{\mathrm{By}}$. Considering Eqs. (13) and (A.16), the first term of Eq. (14) is expressed as

$$
\begin{array}{r}
\frac{1-\frac{n_{\mathrm{B}} \mathbf{T}_{\mathrm{B}} \cdot \mathbf{v}_{\mathrm{B}}}{c}}{1-\frac{n_{\mathrm{A}} \mathbf{T}_{\mathrm{A}} \cdot \mathbf{v}_{\mathrm{A}}}{c}}=\frac{1-\frac{\mathbf{N}_{\mathrm{AB}} \cdot \mathbf{v}_{\mathrm{B}}}{c}-\frac{\left(\mathbf{T}_{\mathrm{B}}-\mathbf{N}_{\mathrm{AB}}\right) \cdot \mathbf{v}_{\mathrm{B}}}{c}-\frac{\left(n_{\mathrm{B}}-1\right) \mathbf{T}_{\mathrm{B}} \cdot \mathbf{v}_{\mathrm{B}}}{c}}{1-\frac{\mathbf{N}_{\mathrm{AB}} \cdot \mathbf{v}_{\mathrm{A}}}{c}-\frac{\left(\mathbf{T}_{\mathrm{A}}-\mathbf{N}_{\mathrm{AB}}\right) \cdot \mathbf{v}_{\mathrm{A}}}{c}-\frac{\left(n_{\mathrm{A}}-1\right) \mathbf{T}_{\mathrm{A}} \cdot \mathbf{v}_{\mathrm{A}}}{c}} \\
\times \frac{1-\frac{\mathbf{N}_{\mathrm{AB}} \cdot \mathbf{v}_{\mathrm{B}}}{c}+\frac{v_{\mathrm{BX}} \delta_{\mathrm{B}} \sin \gamma_{\mathrm{B}}-v_{\mathrm{By}} \delta_{\mathrm{B}} \cos \gamma_{\mathrm{B}}}{c}-\frac{\left(M_{1}+M_{2}\right) \mathbf{N}_{\mathrm{AB}} \cdot \mathbf{v}_{\mathrm{B}}}{c}}{1-\frac{\mathbf{N}_{\mathrm{AB}} \cdot \mathbf{v}_{\mathrm{A}}}{c}-\frac{v_{\mathrm{Ax}} \delta_{\mathrm{A}} \sin \gamma_{\mathrm{B}}-v_{\mathrm{Ay}} \delta_{\mathrm{A}} \cos \gamma_{\mathrm{B}}}{c}+\frac{40.3 n_{\mathrm{e}} \mathbf{N}_{\mathrm{AB}} \cdot \mathbf{v}_{\mathrm{A}}}{c f^{2}}} .
\end{array}
$$

Here, $v_{\mathrm{Ax}}, v_{\mathrm{Ay}}, v_{\mathrm{Bx}}$ and $v_{\mathrm{By}}$ are derived from the components of velocities in LLCS, which are determined by (A.17) and (A.18).

\section{Appendix B}

Considering ACES links $f_{1}$ and $f_{2}$, we mark the positions of the ground station at $t_{1}$ as $B^{\prime}$, space station at $t_{2}$ as $A^{\prime}$, space station at $t_{3}$ as $A$, and ground station at $t_{5}$ as $B$ (as Fig. 2 shows). 
To express the Doppler frequency shift to the order of $c^{-1}$, it is easy to obtain the shift of two links as

$$
\begin{aligned}
& \delta f_{\mathrm{dop} 1}=\left(1-\frac{\mathbf{N}_{\mathrm{B}^{\prime} \mathrm{A}^{\prime}} \cdot \mathbf{v}_{\mathrm{A}^{\prime}}}{c}\right) /\left(1-\frac{\mathbf{N}_{\mathrm{B}^{\prime} \mathrm{A}^{\prime}} \cdot \mathbf{v}_{\mathrm{B}^{\prime}}}{c}\right) \\
& \delta f_{\mathrm{dop} 2}=\left(1-\frac{\mathbf{N}_{\mathrm{AB}} \cdot \mathbf{v}_{\mathrm{B}}}{c}\right) /\left(1-\frac{\mathbf{N}_{\mathrm{AB}} \cdot \mathbf{v}_{\mathrm{A}}}{c}\right) .
\end{aligned}
$$

Considering that the time interval of this process is very short (known for the height of ISS), which is approximately $T_{12}=T_{35}=1 \mathrm{~ms}$; according to [21], we need $T_{23}<1 \mu \mathrm{s}$, two Doppler frequency shifts are numerically close.

To simplify the overall frequency shift, we must express all terms at time $t_{1}$ and $t_{2}$ by the terms at time $t_{3}$ and $t_{5}$. For this study, we deduced the algorithm adopted by the appendix of [23]. Using this algorithm, we have

$$
\begin{aligned}
& \mathbf{N}_{\mathrm{B}^{\prime} \mathrm{A}^{\prime}}=\left(-\mathbf{N}_{\mathrm{AB}}+\frac{\mathbf{r}_{\mathrm{A}^{\prime}}-\mathbf{r}_{\mathrm{A}}}{R_{\mathrm{AB}}}-\frac{\mathbf{r}_{\mathrm{B}^{\prime}}-\mathbf{r}_{\mathrm{B}}}{R_{\mathrm{AB}}}\right) \frac{R_{\mathrm{AB}}}{R_{\mathrm{A}^{\prime} \mathrm{B}^{\prime}}} \\
& \mathbf{r}_{\mathrm{A}^{\prime}}-\mathbf{r}_{\mathrm{A}}=-\mathbf{v}_{\mathrm{A}} T_{23}+\frac{1}{2} \mathbf{a}_{\mathrm{A}} T_{23}^{2} \\
& \mathbf{r}_{\mathrm{B}^{\prime}}-\mathbf{r}_{\mathrm{B}}=-\mathbf{v}_{\mathrm{B}} T_{15}+\frac{1}{2} \mathbf{a}_{\mathrm{B}} T_{15}^{2} \\
& T_{15}=\frac{2 R_{\mathrm{AB}}}{c}-\frac{2 \mathbf{R}_{\mathrm{AB}} \cdot \mathbf{v}_{\mathrm{B}}}{c^{2}}+T_{23} .
\end{aligned}
$$

According to the actual value, we roughly calculated the speed value of ISS, and $v / c$ is approximately $10^{-5}$. Based on this magnitude, the magnitude of $T_{23}$ is approximately nanosecond; thus, they can be considered a $c^{-2}$ term. However, $T_{15}$ is considered a $c^{-1}$ term according to Eq. (B.5). By Eqs. (B.3) and (B.4), neglecting $O\left(c^{-3}\right)$, we can obtain vector $R_{\mathrm{A}^{\prime} \mathrm{B}^{\prime}}$

$\mathbf{R}_{\mathrm{A}^{\prime} \mathrm{B}^{\prime}}=\mathbf{R}_{\mathrm{AB}}+\mathbf{v}_{\mathrm{A}} T_{23}-\mathbf{v}_{\mathrm{B}} T_{15}+\frac{1}{2} \mathbf{a}_{\mathrm{B}} T_{15}^{2}$

After squaring the vector and expanding the root of this result into secondary series, we obtain:

$$
\begin{aligned}
R_{\mathrm{A}^{\prime} \mathrm{B}^{\prime}}= & R_{\mathrm{AB}}-\frac{\left(\mathbf{R}_{\mathrm{AB}} \cdot \mathbf{v}_{\mathrm{B}}\right) T_{15}}{R_{\mathrm{AB}}}+\frac{\left(\mathbf{R}_{\mathrm{AB}} \cdot \mathbf{a}_{\mathrm{B}}\right) T_{15}^{2}}{2 R_{\mathrm{AB}}}+\frac{v_{\mathrm{B}}^{2} T_{15}^{2}}{2 R_{\mathrm{AB}}} \\
& +\frac{\left(\mathbf{R}_{\mathrm{AB}} \cdot \mathbf{v}_{\mathrm{A}}\right) T_{23}}{R_{\mathrm{AB}}}-\frac{\left(\mathbf{R}_{\mathrm{AB}} \cdot \mathbf{v}_{\mathrm{B}}\right)^{2} T_{15}^{2}}{2 R_{\mathrm{AB}}^{3}} .
\end{aligned}
$$

Substitute $T_{15}$ with (B.5), we have

$$
\begin{aligned}
R_{\mathrm{A}^{\prime} \mathrm{B}^{\prime}}= & R_{\mathrm{AB}}-\frac{2 \mathbf{R}_{\mathrm{AB}} \cdot \mathbf{v}_{\mathrm{B}}}{c}+\frac{2\left(\mathbf{R}_{\mathrm{AB}} \cdot \mathbf{a}_{\mathrm{B}}\right) R_{\mathrm{AB}}}{c^{2}}+\frac{2 v_{\mathrm{B}}^{2} R_{\mathrm{AB}}}{c^{2}} \\
& -\frac{\mathbf{R}_{\mathrm{AB}} \cdot\left(\mathbf{v}_{\mathrm{B}}-\mathbf{v}_{\mathrm{A}}\right)}{R_{\mathrm{AB}}} T_{23}
\end{aligned}
$$

and

$$
\begin{aligned}
\frac{R_{\mathrm{AB}}}{R_{\mathrm{A}^{\prime} \mathrm{B}^{\prime}}} & =1-\frac{R_{\mathrm{A}^{\prime} \mathrm{B}^{\prime}}-R_{\mathrm{AB}}}{R_{\mathrm{AB}}}+\left(\frac{R_{\mathrm{A}^{\prime} \mathrm{B}^{\prime}}-R_{\mathrm{AB}}}{R_{\mathrm{AB}}}\right)^{2} \\
& =1+\frac{2 \mathbf{N}_{\mathrm{AB}} \cdot \mathbf{v}_{\mathrm{B}}}{c}-\frac{2\left(\mathbf{N}_{\mathrm{AB}} \cdot \mathbf{a}_{\mathrm{B}}\right) R_{\mathrm{AB}}}{c^{2}}-\frac{2 v_{\mathrm{B}}^{2}}{c^{2}}
\end{aligned}
$$

$$
+\frac{\mathbf{N}_{\mathrm{AB}} \cdot\left(\mathbf{v}_{\mathrm{B}}-\mathbf{v}_{\mathrm{A}}\right)}{R_{\mathrm{AB}}} T_{23}+\frac{4\left(\mathbf{N}_{\mathrm{AB}} \cdot \mathbf{v}_{\mathrm{B}}\right)^{2}}{c^{2}} .
$$

From (B.2)-(B.4) and (B.9), we obtain

$$
\begin{aligned}
\mathbf{N}_{\mathrm{B}^{\prime} \mathrm{A}^{\prime}=} & -\mathbf{N}_{\mathrm{AB}}\left[1+\frac{2 \mathbf{N}_{\mathrm{AB}} \cdot \mathbf{v}_{\mathrm{B}}}{c}-\frac{2\left(\mathbf{N}_{\mathrm{AB}} \cdot \mathbf{a}_{\mathrm{B}}\right) R_{\mathrm{AB}}}{c^{2}}\right. \\
& \left.-\frac{2 v_{\mathrm{B}}^{2}}{c^{2}}+\frac{\mathbf{N}_{\mathrm{AB}} \cdot\left(\mathbf{v}_{\mathrm{B}}-\mathbf{v}_{\mathrm{A}}\right)}{R_{\mathrm{AB}}} T_{23}+\frac{4\left(\mathbf{N}_{\mathrm{AB}} \cdot \mathbf{v}_{\mathrm{B}}\right)^{2}}{c^{2}}\right] \\
& +\frac{2}{c} \mathbf{v}_{\mathrm{B}}\left(1+\frac{\mathbf{N}_{\mathrm{AB}} \cdot \mathbf{v}_{\mathrm{B}}}{c}\right)+\frac{T_{23}}{R_{\mathrm{AB}}}\left(\mathbf{v}_{\mathrm{B}}-\mathbf{v}_{\mathrm{A}}\right)-\frac{2}{c^{2}} R_{\mathrm{AB}} \mathbf{a}_{\mathrm{B}} .
\end{aligned}
$$

With the velocity of the ground station and space station, in terms of velocity, acceleration and derivative of acceleration [23], we have

$\mathbf{v}_{\mathrm{A}^{\prime}}=\mathbf{v}_{\mathrm{A}}-\mathbf{a}_{\mathrm{A}} T_{23}$

$\mathbf{v}_{\mathrm{B}^{\prime}}=\mathbf{v}_{\mathrm{B}}-\frac{2}{c} R_{\mathrm{AB}} \mathbf{a}_{\mathrm{B}}+\frac{2}{c^{2}}\left[\left(R_{\mathrm{AB}} \cdot v_{\mathrm{B}}\right) \mathbf{a}_{\mathrm{B}}+R_{\mathrm{AB}} \mathbf{b}_{\mathrm{B}}\right]-\mathbf{a}_{\mathrm{B}} T_{23}$.

For the approximation of ACES: $\left|\mathbf{N}_{\mathrm{AB}} \cdot \mathbf{v}_{\mathrm{A}} / c\right| \leq 2.6 \times 10^{-5}$, $\left|\mathbf{N}_{\mathrm{AB}} \cdot \mathbf{v}_{\mathrm{B}} / c\right| \leq 1.6 \times 10^{-6},\left|\mathbf{R}_{\mathrm{AB}} \cdot \mathbf{a}_{\mathrm{A}} / c^{2}\right| \leq 1.7 \times 10^{-10}$, and $\left|\mathbf{R}_{\mathrm{AB}} \cdot \mathbf{a}_{\mathrm{B}} / c^{2}\right| \leq 7 \times 10^{-13}$ [23]; to the order of $10^{-16}$, we have

$$
\begin{aligned}
1 & -\frac{\mathbf{N}_{\mathrm{B}^{\prime} \mathrm{A}^{\prime}} \cdot \mathbf{v}_{\mathrm{A}^{\prime}}}{c}=1+\frac{\mathbf{N}_{\mathrm{AB}} \cdot \mathbf{v}_{\mathrm{A}}}{c}+\frac{2\left(\mathbf{N}_{\mathrm{AB}} \cdot \mathbf{v}_{\mathrm{A}}\right)\left(\mathbf{N}_{\mathrm{AB}} \cdot \mathbf{v}_{\mathrm{B}}\right)}{c^{2}} \\
& -\frac{2 \mathbf{v}_{\mathrm{A}} \cdot \mathbf{v}_{\mathrm{B}}}{c^{2}}+\left[\frac{\mathbf{N}_{\mathrm{AB}} \cdot\left(\mathbf{v}_{\mathrm{B}}-\mathbf{v}_{\mathrm{A}}\right)\left(\mathbf{N}_{\mathrm{AB}} \cdot \mathbf{v}_{\mathrm{A}}\right)}{c R_{\mathrm{AB}}}\right. \\
& \left.-\frac{\left(\mathbf{v}_{\mathrm{B}}-\mathbf{v}_{\mathrm{A}}\right) \cdot \mathbf{v}_{\mathrm{A}}}{c R_{\mathrm{AB}}}-\frac{\mathbf{N}_{\mathrm{AB}} \cdot \mathbf{a}_{\mathrm{A}}}{c}\right] T_{23} \\
1 & -\frac{\mathbf{N}_{\mathrm{B}^{\prime} \mathrm{A}^{\prime}} \cdot \mathbf{v}_{\mathrm{B}^{\prime}}}{c}=1+\frac{\mathbf{N}_{\mathrm{AB}} \cdot \mathbf{v}_{\mathrm{B}}}{c}+\frac{2\left(\mathbf{N}_{\mathrm{AB}} \cdot \mathbf{v}_{\mathrm{B}}\right)^{2}}{c^{2}} \\
& -\frac{2 v_{\mathrm{B}}^{2}}{c^{2}}-\frac{2 R_{\mathrm{AB}} \mathbf{N}_{\mathrm{AB}} \cdot \mathbf{a}_{\mathrm{B}}}{c^{2}} \\
& +\left[\frac{\mathbf{N}_{\mathrm{AB}} \cdot\left(\mathbf{v}_{\mathrm{B}}-\mathbf{v}_{\mathrm{A}}\right)\left(\mathbf{N}_{\mathrm{AB}} \cdot \mathbf{v}_{\mathrm{B}}\right)}{c R_{\mathrm{AB}}}-\frac{\left(\mathbf{v}_{\mathrm{B}}-\mathbf{v}_{\mathrm{A}}\right) \cdot \mathbf{v}_{\mathrm{B}}}{c R_{\mathrm{AB}}}\right] T_{23} .
\end{aligned}
$$

\section{References}

1. A. Einstein, Sitzungsber. Preuss. Akad. Wiss. 47(2), 831-839 (1915)

2. A.S. Eddington, Observatory 42, 119 (1919)

3. R.V. Pound, G.A. Rebka, Phys. Rev. Lett. 4(6), 274 (1960). https:// doi.org/10.1103/PhysRevLett.4.274

4. R.V. Pound, J.L. Snider, Phys. Rev. 140(3B), B788 (1965). https:// doi.org/10.1103/PhysRev.140.B788

5. J.C. Hafele, R.E. Keating, Science 177, 168 (1972)

6. J.W. Brault, The gravitational red shift in the solar spectrum. Ph.D. thesis, Princeton University (1962)

7. T.P. Krisher, D.D. Morabito, J.D. Anderson, Phys. Rev. Lett. 70(15), 2213 (1993). https://doi.org/10.1103/PhysRevLett.70. 2213

8. R. Vessot, M. Levine, Gen. Relativ. Gravit. 10(3), 181 (1979) 
9. R.F.C. Vessot, M.W. Levine, E.M. Mattison, E.L. Blomberg, T.E. Hoffman, G.U. Nystrom, B.F. Farrel, R. Decher, P.B. Eby, C.R. Baugher, J.W. Watts, D.L. Teuber, F.D. Wills, Phys. Rev. Lett. 45(26), 2081 (1980). https://doi.org/10.1103/PhysRevLett. 45.2081

10. P. Delva, N. Puchades, E. Schonemann, F. Dilssner, C. Courde, S. Bertone, F. Gonzalez, A. Hees, C. Le Poncin-Lafitte, F. Meynadier, R. Prieto-Cerdeira, B. Sohet, J. Ventura-Traveset, P. Wolf, Phys. Rev. Lett. 121(23), 231101 (2018). https://doi. org/10.1103/PhysRevLett.121.231101. https://www.ncbi.nlm.nih. gov/pubmed/30576203

11. S. Herrmann, F. Finke, M. Lulf, O. Kichakova, D. Puetzfeld, D. Knickmann, M. List, B. Rievers, G. Giorgi, C. Gunther, H. Dittus, R. Prieto-Cerdeira, F. Dilssner, F. Gonzalez, E. Schonemann, J. Ventura-Traveset, C. Lammerzahl, Phys. Rev. Lett. 121(23), 231102 (2018). https://doi.org/10.1103/PhysRevLett.121.231102. https://www.ncbi.nlm.nih.gov/pubmed/30576165

12. B. Altschul, Q.G. Bailey, L. Blanchet, K. Bongs, P. Bouyer, L. Cacciapuoti, S. Capozziello, N. Gaaloul, D. Giulini, J. Hartwig et al., Adv. Space Res. 55(1), 501 (2015)

13. L. Cacciapuoti, C. Salomon, Eur. Phys. J. Spec. Top. 172(1), 57 (2009). https://doi.org/10.1140/epjst/e2009-01041-7

14. L. Cacciapuoti, C. Salomon, J. Phys. Conf. Ser. 327, 012049 (2011). https://doi.org/10.1088/1742-6596/327/1/012049

15. L. Cacciapuoti, P. Laurent, C. Salomon, in Part of Proceedings, 52nd Rencontres de Moriond on Gravitation (Moriond Gravitation 2017), La Thuile, Italy, 25 Mar-1 Apr 2017, pp. 139-148. https:// inspirehep.net/literature/1671213

16. L. Cacciapuoti, M. Armano, R. Much, O. Sy, A. Helm, M.P. Hess, J. Kehrer, S. Koller, T. Niedermaier, F.X. Esnault et al., Eur. Phys. J. D 74(8), 1 (2020)

17. F. Meynadier, P. Delva, C. le Poncin-Lafitte, C. Guerlin, P. Wolf, Class. Quantum Gravity 35(3), 035018 (2018). https://doi.org/10. 1088/1361-6382/aaa279

18. M.P. Hess, J. Kehrer, M. Kufner, S. Durand, G. Hejc, H. Frühauf, L. Cacciapuoti, R. Much, R. Nasca, in ACES MWL status and test results. 2011 Joint Conference of the IEEE International Frequency Control and the European Frequency and Time Forum (FCS) Proceedings (2011), pp. 1-8. https://doi.org/10.1109/FCS. 2011.5977727

19. Y. Guo, in A precise time synchronization method between satellite and ground and its analysis of motion delay error. The 12th China Satellite Navigation Conference. CSNC 2021 (2021)

20. W. Wang, in Precise orbit determination of space station based on GNSS measurement. The 12th China Satellite Navigation Conference. CSNC 2021 (2021)

21. L. Duchayne, F. Mercier, P. Wolf, Astron. Astrophys. 504(2), 653 (2009). https://doi.org/10.1051/0004-6361/200809613

22. E. Savalle, C. Guerlin, P. Delva, F. Meynadier, C. le Poncin-Lafitte, P. Wolf, Class. Quantum Gravity 36(24), 245004 (2019)

23. L. Blanchet, C. Salomon, P. Teyssandier, P. Wolf, Astron. Astrophys. 370(1), 320 (2001). https://doi.org/10.1051/0004-6361: 20010233

24. G.H. Millman, M.C. Arabadjis, Tropospheric and ionospheric phase perturbations and Doppler frequency shift effects. Report, Pasadena, Calif: National Aeronautics and Space Administration, Jet Propulsion Laboratory, California Institute of Technology (1984)
25. G.A. Hajj, L.J. Romans, Radio Sci. 33(1), 175 (1998). https://doi. org/10.1029/97RS03183

26. K. Davies, J.M. Watts, D.H. Zacharisen, J. Geophys. Res. 67, 601 (1962). https://doi.org/10.1029/JZ067i002p00601. https://ui. adsabs.harvard.edu/abs/1962JGR...67..601D

27. G. Petit, B. Luzum, IERS Conventions. Report, Bureau International des Poids et Mesures Sevres (France) (2010)

28. K. Davies, D.M. Baker, Radio Sci. 1(5), 545 (1966). https://doi. org/10.1002/rds 196615545

29. J.A. Jacobs, T. Watanabe, Radio Sci. 1(3), 257 (1966). https://doi org/10.1002/rds196613257

30. Z. Shen, W.B. Shen, S. Zhang, Geophys. J. Int. 206(2), 1162 (2016). https://doi.org/10.1093/gji/ggw198

31. J.A. Bennett, Aust. J. Phys. 21, 259 (1968). https://doi.org/10. 1071/PH680259. https://ui.adsabs.harvard.edu/abs/1968AuJPh.. 21..259B

32. K. Davies, Ionospheric Radio Propagation, vol. 80 (US Department of Commerce, National Bureau of Standards, Gaithersburg, 1965)

33. W.G. Melbourne, E.S. Davis, C.B. Duncan, G.A. Hajj, K.R. Hardy, E.R. Kursinski, T.K. Meehan, L.E. Young, T.P. Yunck, in The application of spaceborne GPS to atmospheric limb sounding and global change monitoring (National Aeronautics and Space Administration, Jet Propulsion Laboratory, California Institute of Technology, 1994). https://ntrs.nasa.gov/api/citations/ 19960008694/downloads/19960008694.pdf

34. F. Meynadier, P. Delva, C. le Poncin-Lafitte, C. Guerlin, P. Laurent, P. Wolf, in ACES MWL data analysis center at SYRTE. SF2A2017: Proceedings of the Annual meeting of the French Society of Astronomy and Astrophysics (2017). https://ui.adsabs.harvard.edu/ abs/2017sf2a.conf..365M

35. C.M. Will, Living Rev. Relativ. 17(1), 4 (2014). https://doi. org/10.12942/lrr-2014-4. https://www.ncbi.nlm.nih.gov/pubmed/ 28179848

36. M.M. Hoque, N. Jakowski, Radio Sci. 43, 5 (2008). https://doi.org/ $10.1029 / 2007 \mathrm{rs} 003817$

37. J. Wahr, Global Earth Physics. AGU Reference Shelf, vol. 1 (1995), p. 40. https://doi.org/10.1029/RF001p0040

38. C. Voigt, H. Denker, L. Timmen, Metrologia 53(6), 1365 (2016). https://doi.org/10.1088/0026-1394/53/6/1365

39. D.F. Argus, R.G. Gordon, Geophys. Res. Lett. 18(11), 2039 (1991). https://doi.org/10.1029/91GL01532

40. T. Hartmann, H. Wenzel, Geophys. Res. Lett. 22(24), 3553 (1995)

41. N. Pavlis, S. Holmes, S. Kenyon, J. Factor, in The EGM 2008 global gravitational model. AGU Fall Meeting Abstracts (2008)

42. D.W. Allan, M.A. Weiss, J.L. Jespersen, in A frequency-domain view of time-domain characterization of clocks and time and frequency distribution systems. Proceedings of the 45th Annual Symposium on Frequency Control 1991 (1991), pp. 667-678. https:// doi.org/10.1109/FREQ.1991.145966

43. L. Galleani, L. Sacerdote, P. Tavella, C. Zucca, Metrologia 40(3), S257 (2003). https://doi.org/10.1088/0026-1394/40/3/305

44. N.J. Kasdin, Proc. IEEE 83(5), 802 (1995). https://doi.org/10. $1109 / 5.381848$

45. T.A. Croft, H. Hoogansian, Radio Sci. 3(1), 69 (1968). https://doi. org/10.1002/rds 19683169 